\title{
Zum Einfluss von Fallbeispielen und furchterregenden Bildern auf die Wirksamkeit von Gesundheitsflyern zum Thema Adipositas"
}

\author{
Constanze Rossmann / Tanja Pfister
}

Die Frage, wie Gesundheitsinformationen optimal aufbereitet werden, um die Bevölkerung zu erreichen, ist eines der zentralen Problemfelder der Gesundheitskommunikation. Eine bäufig eingesetzte Strategie sind Furchtappelle, über deren gesundheitsförderliche Wirkung bis dato widersprüchliche Ergebnisse vorliegen. Auch Fallbeispiele können die Wabrnebmung gesundheitsspezifischer Risiken, Einstellungen und Verbaltensintentionen beeinflussen. Die Richtung der Einflüsse und ob furchterregende Bilder mit Fallbeispielen interagieren, ist bislang nicht eindeutig geklärt. Wir fübrten ein $2 \times 3$-Experiment mit zweimaliger Nachbermessung zu Informationsflyern über Adipositas durch (Faktor 1: Fallbeispiel vs. summarische Realitätsbeschreibung, Faktor 2: positives, leicht negatives, sebr negatives Bild). Die erste Nachbermessung $(n=185)$ erbob intervenierende (Soziodemographie, Involvement, Flyerbewertung etc.) und abbängige Variablen (Wissen, Risikowabrnebmung, Einstellungen, Verbaltensintention), die zweite erfasste die abhängigen Variablen 14 Tage später erneut und zudem das Verbalten wäbrend der vergangenen zwei Wochen $(n=176)$. Die Ergebnisse zeigen keine Unterschiede durch die Textversion. Die Bilder hingegen beeinflussten das Wissen in Form einer U-Funktion sowie Risikowabrnebmung, Einstellungen und Verbaltensintentionen - wenn auch nur teilweise signifikant - in Form einer umgekehrten U-Funktion. Beim Verhalten war die Wirkung des leicht negativen und des negativen Bildes am stärksten. Die Befunde waren größtenteils unabhängig von intervenierenden Merkmalen und der Einfluss des Flyers blieb über die zwei Wochen hinweg weitgehend stabil.

Schlagwörter: Gesundheitskommunikation, Informationsflyer, Adipositas, Fallbeispiele, Furchtappelle, furchterregende Bilder

\section{Einleitung}

Die Frage, wie Gesundheitsinformationen optimal aufbereitet werden und welche Informationskanäle sich eignen, um die Bevölkerung zu erreichen, ist eines der zentralen Problemfelder der Gesundheitskommunikation. Als Bereich der Kommunikationswissenschaft fand die Gesundheitskommunikation in Deutschland bislang eher geringe Beachtung, während sie unter dem Begriff „health communication“ in den USA schon seit etwa 35 Jahren eine etablierte Disziplin ist (Jazbinsek 2000). Neben interpersonaler Kommunikation werden vor allem direkt invasive Eingriffe als wirkungsvolle Strategien der Gesundheitsförderung beschrieben (z. B. Welch Cline 1990, Cegala 2007). Interpersonale Förderung ist aber sowohl kosten- als auch personalintensiv. Ein Ressourcen sparender Weg, um Menschen gesundheitsförderliche Informationen nahezubringen, sind neben den klassischen massenmedialen Informationskanälen wie Presse, Rundfunk oder Plakatwerbung auch Gesundheitsflyer. Gesundheitsflyer sind in der Regel keine

* Die Studie entstand mit finanzieller Unterstützung durch das Münchner Zentrum für Gesundheitswissenschaften (MC-Health) der Ludwig-Maximilians-Universität München. 
für sich isolierten Kommunikationsmittel, sondern gelten vielmehr als fester Bestandteil von Kommunikationskampagnen (Gregory 2002). ${ }^{1}$ Sie präsentieren auf wenigen Seiten verdichtet Informationen über spezifische Krankheitsbilder, ihre Ursachen, Folgen und Präventionsmöglichkeiten. Sie werden in hohen Auflagen produziert und variieren enorm (Gregory 2002). So gibt es „unzählig viele Broschüren zu einem breiten Spektrum an Gesundheitsthemen“" (Naidoo \& Wills 2003: 253). In der Literatur werden jedoch keine exakten Daten genannt, weder zu genauen Auflagenzahlen noch über das speziell für Flyer aufgewendete Budget. Auch wenn die Bundeszentrale für gesundheitliche Aufklärung (BZgA) allein zum Thema "Gesundheitsförderung von Kindern und Jugendlichen" bis zu 250.000 Flyer und Broschüren jährlich verteilt (BZgA 2007), sagt das noch wenig über das Kostenverhältnis von Gesundheitsflyern im Verhältnis zur gesamten Kampagne aus. Insgesamt nutzen Organisationen Printmaterial jedoch häufiger als andere Kommunikationsmittel (PR News: o.V. 1997), was auch für einen hohen Anteil an Flyern spricht. Aus Sicht der Rezipienten sind Gesundheitsflyer die am häufigsten genutzte Quelle für medizinische Informationen (Närhi 2007). Patienten sehen in ihnen hilfreiche Mittel, um Ängste zu reduzieren, Vertrauen und Motivation zu steigern, medizinischen Rat einzuholen; zudem helfen sie, das Wissen zu steigern (Hawkey \& Hawkey 1989, Kai 1994). Insgesamt scheinen Gesundheitsflyer also ein wichtiges und gebräuchliches Mittel der Gesundheitsprävention. Allerdings zeigen verschiedene Studien, dass ihre Wirksamkeit begrenzt ist. So fand Hunt (1995) heraus, dass Flyer zur Ernährung nicht angemessen eingesetzt und daher häufig gar nicht gelesen wurden (vgl. auch Nichols et al. 1988). Auch Glanz (1985) und Thomas (1994) belegten, dass Flyer im Ernährungskontext nicht geeignet sind, um Informationen zu vermitteln. Es stellt sich also die Frage, wie sich die Wirksamkeit von Gesundheitsflyern verbessern lässt, konkret, welchen Einfluss bestimmte Gestaltungsmittel auf die Wirksamkeit von Gesundheitsflyern haben.

\section{Hintergrund}

\subsection{Furchtappelle und furchterregende Bilder}

Ein häufig eingesetztes Mittel zur Verdeutlichung der Risiken gesundheitsschädlichen Verhaltens ist der Furchtappell. Dieser taucht entweder in Form von besonders drastischen Formulierungen im Text auf (z. B. schlimmstmögliche Krankheitsfolgen) oder in Form von besonders negativ anmutenden Bildern. Die bisherige Forschung ist sich noch uneins, ob der Einsatz von Furchtappellen die Wirksamkeit von Gesundheitsinformationen positiv oder negativ beeinflusst (Friend \& Levy 2002). Die Ausgangshypothese von Hovland, Janis \& Kelley (1953) propagierte: Je furchterregender eine Botschaft, desto effektiver der Furchtappell. Janis \& Feshbach (1953) fanden in ihrer Pionierstudie zum Einfluss von Furchtappellen auf die Wirksamkeit von Gesundheitsbotschaften über Zahnpflege dagegen einen negativen Zusammenhang: Je stärker der Furchtappell, desto weniger putzten die Probanden hinterher ihre Zähne. Ähnliche Ergebnisse fanden Krisher, Darley \& Darley (1973). Sie zeigten, dass Probanden, die einem moderaten Furchtappell ausgesetzt waren, sich eher einer Mumpsimpfung unterzogen als Probanden, die einen sehr starken (oder sehr schwachen) Furchtappell rezipierten. Die kon-

1 Auch Bonfadelli \& Friemel (2006) betonen den bei Gesundheitskampagnen genutzten „Mediamix“, der sich aus „paid media“, „free media“, Medien-Events und interpersonaler Kommunikation zusammensetzt. 
traproduktive Wirkungsweise von Furchtappellen beschreiben auch Kohn, Goodstadt, Cook, Sheppard \& Chan (1982), die in ihrer Studie den Einfluss abschreckender Filme zum Thema Verkehrsunfälle untersuchten. Sie präsentierten ihren Probanden den Film „Collision Course“ in drei unterschiedlichen Versionen: Einmal als Beinahezusammenstoß zweier Fahrzeuge (niedrige Bedrohung), einmal in Form eines schweren Verkehrsunfalls, bei dem zwei Menschen sterben, die Folgen am Fahrzeug und die Toten jedoch nicht gezeigt werden (mittlere Bedrohung), und einmal als Darstellung des schweren Verkehrsunfalls mit sichtbaren und hörbaren Folgen des Unfalls. Die Furchtappelle erzielten nicht die erwünschten Einflüsse auf Einstellungen und Verhalten. Vielmehr führte der starke (oder sehr schwache) Furchtappell zum sog. „Boomerang Effekt“: Anstatt Alkohol am Steuer abzulehnen, waren die Probanden nach der Rezeption des Furchtappells demgegenüber signifikant positiver eingestellt. Einen solchen kurvilinearen Zusammenhang zwischen Furcht und Persuasion hatte schon Miller (1963) vorgeschlagen.

Einige Studien und Meta-Analysen deuten hingegen darauf hin, dass Furchtappelle einen positiven Einfluss auf die Effektivität von Gesundheitsbotschaften haben, und stimmen damit v. a. mit Hovland et al. (1953) überein. Ein stärkerer Furchtappell würde demnach zu stärkeren Einstellungs-, Verhaltensintentions- und Verhaltensänderungen führen. Andere Studien deuten eher auf einen negativen Zusammenhang von Furchtappell und der Durchführung des empfohlenen Verhaltens hin, wiederum andere bestätigen Millers (1963) Hypothese eines kurvilinearen Zusammenhangs (Sutton 1982, Boster \& Mongeau 1984, Mongeau 1998, Witte \& Allen 2000, Devos-Comby \& Salovey 2002, Green \& Witte 2006). Heute geht man davon aus, dass sich die Effektivität des Furchtappells vermutlich am besten als umgekehrte U-Funktion beschreiben lässt (vgl. Vögele 2007): Ein leichter Furchtappell scheint die Wirksamkeit von Gesundheitsinformationen positiv zu beeinflussen, während sehr starke Furchtappelle und Informationen ohne Furchtappell weniger effektiv sind. Es muss jedoch darauf hingewiesen werden, dass die Wirkung von Furchtappellen stark von den Prädispositionen der Rezipienten abhängt. So sieht Witte (1992) drei zentrale Konstrukte eines Furchtappells: Furcht, Bedrohung und Selbstwirksamkeit. Von herausragender Bedeutung ist in diesem Zusammenhang die Wahrnehmung dieser drei Komponenten, die von Rezipient zu Rezipient je nach Prädisposition variiert. Die negative Emotion Furcht wird durch eine Bedrohung (z. B. durch ein bedrohliches Bild) geweckt. Im Idealfall sollte der Rezipient die wahrgenommene Furcht als bedeutsam und persönlich relevant ansehen (vgl. auch Easterling \& Leventhal 1989, Ortony \& Turner 1990). Auch die wahrgenommene Schwere der Bedrohung und die wahrgenommene persönliche Bedrohung können bei unterschiedlichen Rezipienten völlig unterschiedlich ausfallen. Von wahrgenommener Furcht und wahrgenommener Bedrohung hängt wiederum die individuell verschiedene Selbstwirksamkeit ab, d. h. die Überzeugung, ein empfohlenes Verhalten ausführen zu können. Diese drei Komponenten beeinflussen sich nicht nur gegenseitig, sondern auch die Akzeptanz der Botschaft und damit die Wirksamkeit des Furchtappells. Sind wahrgenommene Bedrohung und wahrgenommene Wirksamkeit hoch, ist auch die Akzeptanz der Botschaft hoch (Witte 1992). Sind wahrgenommene Bedrohung oder wahrgenommene Selbstwirksamkeit jedoch zu niedrig oder die ausgelöste Furcht zu hoch, können sich die Effekte ins Gegenteil umkehren und die Furchtappelle wirken entweder gar nicht oder schlagen sich sogar in der Förderung des unerwünschten Verhaltens nieder (ebd.).

Für die Wirksamkeit von Bildern mit unterschiedlicher Anmutungsvalenz konnten zum Teil ähnliche Effekte nachgewiesen werden wie für Furchtappelle. So untersuchten 
etwa Zillmann \& Gan (1996) in ihrer Studie zur Wahrnehmung von Gesundheitsrisiken in Nachrichtenbeiträgen die Wirkung bedrohlicher Bilder. Die Probanden sahen nacheinander vier Nachrichtenbeiträge, die sie jeweils sofort beurteilen mussten. Der dritte Beitrag (zum Thema Hautkrebs) war manipuliert: Entweder sahen die Probanden hier relativ harmlose neutrale Bilder oder eher bedrohliche und furchterregende Darstellungen der Krankheit. Die Ergebnisse zeigen, dass sowohl die bedrohlichen als auch die harmlosen Bilder zu einer erhöhten Einschätzung des Erkrankungsrisikos führen. Die Rezipienten der bedrohlichen Bilder waren jedoch stärker beunruhigt als die Rezipienten der harmlosen Bilder. Auch die Bedeutung von Schutzmaßnahmen (z. B. Verwendung von Sonnencremes) schätzten sie höher ein. Zudem zeigte sich eine Interaktion mit dem Geschlecht: Frauen reagierten stärker auf die harmlosen Bilder, während auf Männer eher die bedrohlichen bzw. furchterregenden Bilder wirkten. Durch eine zweite, nicht angekündigte Nachhermessung konnten die Autoren außerdem zeigen, dass der Einfluss fesselnder Bilder mit der Zeit steigt, während der Einfluss von harmlosen Bildern sinkt (ebd.).

Zillmann, Gibson \& Sargent (1999) untersuchten den Einfluss von bedrohlichen Bildern erneut. Den Probanden wurden drei Artikel vorgelegt, einer davon handelte vom Thema "Sicherheit in Vergnügungsparks“. Der Artikel enthielt entweder kein Bild (Kontrollversion), ein Bild, das Achterbahnfahrten als sicher und vergnüglich darstellte (es zeigte fröhliche Jugendliche bei einer Achterbahnfahrt), ein Bild, das die Risiken und Gefahren solcher Achterbahnfahrten präsentierte (es zeigte eine Person auf einer Trage, die in einen Krankenwagen gehoben wird, im Hintergrund ist eine Achterbahn zu sehen), oder beide Bilder. Es stellte sich heraus, dass das bedrohliche Bild einerseits die Einschätzung der allgemeinen Sicherheit beeinflusste bzw. senkte und andererseits die persönliche Sorge um die Sicherheit bei Achterbahnfahrten erhöhte. Der Effekt trat sowohl direkt nach dem Lesen auf als auch bei einer zweiten Befragung, die zehn Tage später stattfand.

Es gibt also deutliche Hinweise darauf, dass Furchtappelle, die in Form von Bildern dargestellt werden, Risikowahrnehmung, Einstellungen und Verhaltensintentionen im Gesundheitsbereich beeinflussen. Die disparate Befundlage zum Einfluss von Furchtappellen generell und der bislang recht dünne Forschungsstand zur quantitativen Messung der Bildwirkung (Petersen 2006) machen es jedoch notwendig, ihren Einfluss erneut $\mathrm{zu}$ untersuchen.

\subsection{Fallbeispiele}

Fallbeispiele, d. h. die Darstellung eines Problems anhand einzelner Personen, sind das zweite Gestaltungsmerkmal, das im Rahmen der vorliegenden Studie untersucht werden soll. Generell sind Fallbeispiele ein bewährtes Mittel, um Informationen lebhaft und anschaulich zu gestalten. Ihre Wirkung, der Fallbeispieleffekt, wird seit Anfang der neunziger Jahre in der Kommunikationswissenschaft untersucht: In der Regel werden Fallbeispiele sog. summarischen Realitätsbeschreibungen, die einen Sachverhalt mit Tatsachen, Zahlen und Fakten abstrakt und systematisch beschreiben, experimentell gegenübergestellt. Wiederholt hat sich gezeigt, dass Fallbeispiele die Wahrnehmung eines Problems, Kausalattributionen und Einstellungen stärker beeinflussen als die eigentlich valideren summarischen Realitätsbeschreibungen (z. B. Brosius \& Bathelt 1994, Brosius 1995, Brosius, Schweiger \& Rossmann 2000, Zillmann \& Brosius 2000, Daschmann 2001, Hoeken \& Hustinx 2007). Keller (2008) konnte nachweisen, dass Fallbeispiele 
auch Verhaltensintentionen - konkret die Spendenbereitschaft von Rezipienten - beeinflussen.

Fallbeispiele dürften sich somit auch für die Vermittlung von Krankheiten und Risiken in der Gesundheitskommunikation eignen. Verschiedene Fallbeispielexperimente, die thematisch im Bereich der Wahrnehmung von Gesundheitsrisiken angesiedelt sind, bestätigen dies. So legten Zillmann, Perkins \& Sundar (1992) ihren Probanden verschiedene Versionen eines Magazinbeitrags vor, die den Erfolg von Diätprogrammen behandelten. Die Beiträge kombinierten summarische Realitätsbeschreibungen mit Fallbeispielen, wobei jeder Beitrag dieselbe summarische Information (ein Drittel der Personen, die die Diät gemacht hatten, hat wieder zugenommen) enthielt. Diese Information wurde zusammen mit unterschiedlichen Fallbeispielverteilungen präsentiert (Version selektiv: alle Fallbeispiele haben wieder zugenommen; Version repräsentativ: ein Drittel hat wieder zugenommen; Version gemischt: die Hälfte hat wieder zugenommen). Die Ergebnisse bestätigen den stärkeren Einfluss der singulären Fallbeispielinformation im Vergleich zur summarischen Realitätsbeschreibung: Die Probanden, die die selektive Version gelesen hatten, überschätzten den Anteil derer, die nach dem Diätprogramm wieder zugenommen hatten, hinterher deutlich. Probanden, die die gemischte Version gelesen hatten, überschätzten den Anteil immer noch leicht. In einer späteren Studie zeigten die Autoren zudem, dass der Einfluss von Fallbeispielen auf die Wahrnehmung der Häufigkeit sozialer Probleme auch über die Zeit hinweg - hier zwei Wochen - stabil waren (Zillmann, Gibson, Sundar \& Perkins 1996).

Aust \& Zillmann (1996) konnten die stärkere Wirkung der Fallbeispiele auch für Fernsehbeiträge nachweisen. Die Autoren manipulierten Nachrichtenbeiträge über die Verbreitung von Salmonellen in einem Restaurant. Die Beiträge lieferten zunächst Fakten zu Personen, die an einer Salmonellenvergiftung litten. Anschließend wurden in zwei Experimentalversionen mehr oder weniger stark emotionalisierte Interviews mit Verwandten der Erkrankten gezeigt, während der Kontrollbeitrag lediglich die (in allen Versionen konstant gehaltenen) Fakten ohne Interviews präsentierte. Beide Fallbeispielversionen (weniger und stark emotionalisierte Interviews) erhöhten das wahrgenommene Risiko, an einer Salmonellenvergiftung zu erkranken, im Vergleich zur Kontrollversion.

Auch Hoeken \& Geurts (2005) untersuchten die Wirksamkeit von Fallbeispielen im Zusammenhang mit gesundheitskommunikativen Inhalten. In ihrer Studie legten sie den Probanden eine zweiseitige Informationsbroschüre zur Gefahr von Internetsucht vor. Sie enthielt das Fallbeispiel einer fiktiven Studentin, die die Entstehung ihrer Internetsucht sowie deren physische und psychische Folgen beschrieb. Den Probanden wurden verschiedene Formen dieser Botschaft vorgelegt. In der einen Version führte die Studentin das empfohlene Gesundheitsverhalten erfolgreich aus und überwand ihre Sucht am Ende. In der anderen Version scheitert die Studentin bei diesem Versuch und bricht daraufhin ihr Studium ab. Das Ergebnis der Studie lässt sich wie folgt zusammenfassen: Die Probanden schätzten die negativen Konsequenzen gravierender und wahrscheinlicher ein, wenn sie durch die erfolgreiche Studentin dargestellt wurden. Außerdem steigerte diese Version die wahrgenommene Selbstwirksamkeit der Probanden sowie ihre Intention, das gesundheitsschädliche Verhalten zu reduzieren. Insgesamt konnte also ein Fallbeispiel, das eine Person darstellt, die das empfohlene Verhalten erfolgreich ausführt, besser überzeugen als ein Fallbeispiel, bei dem die Person in der Umsetzung des empfohlenen Verhaltens scheitert. Streng genommen lässt sich auf Basis dieser Studie zwar nichts darüber aussagen, ob das Fallbeispiel im Vergleich zu rein statistischen Re- 
alitätsbeschreibungen besser wirkt, aber auch diese Studie bestätigt, dass die im Kontext eines Fallbeispiels präsentierten Gesundheitsinformationen generell wirksam sind.

Zillmann (2006) empfiehlt den Einsatz von Fallbeispielen für die wirkungsvolle Vermittlung von Informationen über Gesundheitsrisiken. Die relevanten Erkenntnisse aus der Fallbeispielforschung fasst er wie folgt zusammen: (1) Summarische Realitätsbeschreibungen zur Darstellung gesundheitsspezifischer Risiken sind weitgehend ineffektiv. (2) Relative Krankheitszahlen werden auf Basis der Verteilung der Fallbeispiele wahrgenommen. (3) Die Wahrnehmung des eigenen Gesundheitsrisikos wird aus der Wahrnehmung relativer Krankheitszahlen abstrahiert. (4) Obwohl der selektive Einsatz emotionaler Fallbeispiele die Kriterien repräsentativer Fallbeispielverwendung verletzt, sollten Fallbeispiele eingesetzt werden, um auf Gesundheitsthemen aufmerksam zu machen. (ebd.: S231f.)

Neben Fallbeispielen in Textform plädiert Zillmann (2006) dafür, illustrierende Bilder einzusetzen: "The use of exemplifying imagery, mostly as a complement to informative text, has emerged as a powerful means of creating risk consciousness and of motivating protective and corrective action." (ebd.: S232) Zillmann (2006) verweist in diesem Kontext auf Studien, die weiter oben bereits beschrieben wurden (Zillmann \& Gan 1996, Zillmann, Knobloch \& Yu 2001), und stellt diese als Weiterführung der Fallbeispielforschung dar. Er vermischt damit letztlich zwei Faktoren, die es zu trennen gilt: Fallbeispiele und furchterregende Bilder. Wie oben dargelegt, haben beide Darstellungsmittel auch alleine das Potenzial, die Wahrnehmung von Gesundheitsrisiken, gesundheitsspezifische Einstellungen und Verhaltensintentionen $\mathrm{zu}$ beeinflussen. $\mathrm{Ob}$ das Zusammenspiel der beiden Faktoren die Einflüsse verstärkt, wurde bislang kaum untersucht. Hoeken \& Geurts (2005) verbinden in ihrer Studie zwar quasi Furchtappell und Fallbeispiel miteinander, doch wird die Darstellung der Informationen in Form von Fallbeispielen nicht variiert und somit werden keine Interaktionseffekte zwischen Fallbeispiel und Furchtappell gemessen. Hinweise auf ein solches Zusammenspiel liefert die bereits erwähnte Studie von Keller (2008) im Kontext von Spendenaufrufen. Eine eindeutige Interaktionsrichtung zwischen Fallbeispielen und Bildern lässt sich jedoch bislang nicht ausmachen, weshalb dieses Zusammenspiel hier erneut betrachtet wird.

\subsection{Adipositas}

Thematischer Kontext der Untersuchung ist das Krankheitsbild Adipositas (auch Fettleibigkeit, Fettsucht oder Obesitas). Von Adipositas spricht man, wenn die Körperfettmasse über das normale Maß hinaus erhöht ist. Nach WHO-Definition ist dies ab einem Body-Mass-Index (BMI) von $30,0 \mathrm{~kg} / \mathrm{m}^{2}$ (Verhältnis von Körpergewicht zur quadrierten Körpergröße) der Fall. Übergewicht (auch Präadipositas) liegt bei einem $\mathrm{BMI}$ von 25,0 bis $29,9 \mathrm{~kg} / \mathrm{m}^{2}$ vor (vgl. WHO 2007). Wie in vielen anderen westlichen Industrienationen nimmt die Prävalenz von Übergewicht und Adipositas in Deutschland seit vielen Jahren kontinuierlich zu. Inzwischen sind etwa zwei Drittel der männlichen Bevölkerung und die Hälfte der weiblichen Bevölkerung in Deutschland übergewichtig oder adipös (Robert Koch-Institut 2005). Mit einem durchschnittlichen BMI von $27,3 \mathrm{~kg} / \mathrm{m}^{2}$ liegt Deutschland im internationalen Vergleich im oberen Bereich, nur noch knapp hinter den USA mit 28,1 kg/m² (Wirth 2008: 42). Neben dem Einfluss von Alter und Geschlecht spielen für die Prävalenz von Übergewicht und Adipositas auch Bildung, sozialer Status, Bundesland und Herkunft eine Rolle - Unterschiede, die sich hauptsächlich über den Lebensstil auf das Körpergewicht auswirken (Mensink, Lampert \& Bergmann 2005). Denn als Hauptursachen für Übergewicht und Adipositas 
gelten neben der genetischen Prädisposition eine unausgewogene Ernährung (übermäßige Energieaufnahme, zu viel Fett, zu viel Süßes, zu viel Alkohol und zu wenig Ballaststoffe) und ein bewegungsarmer Alltag der Personen (ebd., Robert Koch-Institut 2005). Übergewicht und Adipositas sind die Hauptursachen für Bluthochdruck, Diabetes Mellitus Typ 2, Fettstoffwechselstörungen und Folgekrankheiten wie Herzinfarkt und Schlaganfall. Das Sterblichkeitsrisiko ist bei übergewichtigen Menschen um 20 bis 40 Prozent, bei adipösen um das zwei- bis dreifache erhöht (vgl. Wirth 2008: 50). Dies verursacht auch Kosten: Allein acht Prozent aller Krankheitskosten gehen inzwischen auf Adipositas zurück (ebd.: 8).

Das Thema Adipositas ist somit nicht nur für Politik und medizinische Versorgung hochrelevant, sondern auch für die Gesundheitsförderung. Die Darstellung von Informationen über Adipositas in Gesundheitsflyern kann sicher nur ein minimaler Baustein einer größer angelegten Präventionskampagne sein. Wie eingangs dargestellt, finden Informationsflyer in der Gesundheitskommunikation jedoch durchaus häufig Anwendung. Die Notwendigkeit, die im Flyer enthaltenen Informationen so zu präsentieren, dass sie möglichst wirksam sind, wird auch vor dem Hintergrund der Prävalenzentwicklung von Adipositas deutlich. Daher wurde dieses Thema für das Experiment ausgewählt.

\subsection{Hypothesen und Forschungsfragen}

Die vorliegende Studie untersucht im Rahmen des Themas Adipositas, inwieweit der Einsatz von Fallbeispielen auf der einen Seite und von Bildern unterschiedlicher Valenz (positiv, leicht negativ, sehr negativ) auf der anderen die Wirksamkeit von Gesundheitsflyern beeinflusst. Aus den bisherigen Befunden zum Fallbeispieleffekt und zum Einfluss furchterregender Bilder leiten wir unsere zentrale Annahme ab: Gesundheitsflyer, die spezifische Krankheitsinformationen anhand eines Fallbeispiels darstellen und mit einem leicht negativen Bild illustrieren, haben einen stärkeren Einfluss auf Wissen, Risikowahrnehmung, Einstellungen, Verhaltensintention und Verhalten als Gesundheitsflyer, die die Informationen in Form von summarischen Realitätsbeschreibungen aufbereiten und mit positiven oder sehr negativen Bildern illustrieren. Konkret lauten unsere Hypothesen und Forschungsfragen wie folgt:

H1: Gesundheitsinformationen, die in Form eines Fallbeispiels dargestellt sind, baben einen stärkeren Einfluss auf a) Wissen, b) Risikowabrnebmung, c) Einstellungen, d) Verbaltensintentionen und e) tatsächliches Verbalten als Gesundheitsinformationen, die in Form von summarischen Realitätsbeschreibungen präsentiert werden.

Studien zum Einfluss von Fallbeispielen belegen wiederholt die Unabhängigkeit des Effektes von Rezipientenmerkmalen (vgl. z. B. Brosius, Schweiger \& Rossmann 2000, im Überblick vgl. Daschmann 2001). Entsprechend postulieren wir:

H2: Der Einfluss des Fallbeispiels im Vergleich zur summarischen Realitätsbeschreibung ist unabhängig von soziodemographischen Merkmalen, der generellen Nutzung von Gesundheitsflyern, Involvement, eigenem Körpergewicht und physischer Aktivität der Rezipienten.

Während die bisherigen Befunde zum Fallbeispieleffekt eine konkrete Hypothese zulassen, sind die Ergebnisse zum Einfluss der Bildvalenz bislang weniger eindeutig. Deshalb formulieren wir hier offene Forschungsfragen: 
F1: Welchen Einfluss hat die Valenz von Bildern auf die Wirksamkeit von Gesundheitsflyern in Bezug auf a) Wissen, b) Risikowabrnebmung, c) Einstellungen, d) Verbaltensintentionen und e) tatsächliches Verbalten?

F2: Welchen Einfluss haben emotionale Reaktionen, soziodemographische Merkmale, Nutzung von Gesundheitsflyern, Involvement, eigenes Körpergerwicht und physische Aktivität der Rezipienten auf den Einfluss der Bildvalenz?

Zillmann (2006) postuliert ein größeres Wirkungspotenzial durch die Verwendung von Fallbeispielen zusammen mit illustrierenden Bildern. Keller (2008) konnte Interaktionseffekte zwischen der Bildvalenz und Fallbeispielen nachweisen, die jedoch keine klare Richtung vorhersagen. Daher lautet unsere Forschungsfrage:

F3: Gibt es einen Interaktionseffekt zwischen der Valenz von Bildern und Fallbeispielen in Bezug auf die Wirksamkeit von Gesundheitsflyern bezüglich a) Wissen, b) Risikowahrnehmung, c) Einstellung, d) Handlungsintention und e) Verhaltensänderung?

Die Befunde zur Stabilität der Einflüsse sind divergent. Davon ausgehend, dass die Probanden die erhaltenen Informationen mit der Zeit schlichtweg vergessen, wäre anzunehmen, dass die Einflüsse des Gesundheitsflyers innerhalb von zwei Wochen geringer werden. Zillmann, Perkins \& Sundar (1992) zeigten in ihrem Experiment, dass der Fallbeispieleffekt auch nach zwei Wochen noch nachzuweisen war, Gibson \& Zillmann (1994) beobachteten nach einer Woche sogar einen stärkeren Fallbeispieleffekt. Zillmann \& Gan (1996) fanden je nach Stimulus unterschiedlich stabile Effekte, die darauf hindeuten, dass der Einfluss der Textversion über die Zeit hinweg eher abnimmt, während der Einfluss des Bildes stabil bleibt (vgl. Zillmann, Gibson \& Sargent 1999, Zillmann 2006). Daher formulieren wir die offene Forschungsfrage:

F4: Wie stabil sind die Einflïsse des Flyers anf Wissen, Risikowabrnebmung und Einstellungen?

\section{Methode und Durchführung}

Um dies zu untersuchen, wurde ein zweifaktorielles Experiment mit zweimaliger Nachhermessung durchgeführt. Als Stimulus diente ein von uns entworfener Informationsflyer zum Thema Adipositas (Fettleibigkeit), der allgemeine Informationen zu Definition und Verbreitung von Adipositas enthielt, über Ursachen und Folgen aufklärte und Hinweise zur Vermeidung der Erkrankung lieferte. Der Flyer wies das gängige, zweimal gefaltete DIN A4-Format auf (vgl. Gregory 2002: 68). Auf der Vorderseite war eine Waage mit dem Fuß eines Menschen abgebildet. Darunter standen die Worte „Adipositas - Fakten | Risiken | Chancen“ sowie ein erfundenes Logo und ein ebenfalls fiktiver Name der „Gesellschaft für Ernährung und Gesundheit“. Logo und Name der Gesellschaft fanden sich auch auf der Rückseite des Flyers, ergänzt um eine fiktive Internet- und Postanschrift. Innen wurden zunächst Verhaltenstipps aufgeführt (Rubriken „Prüfen Sie Ihre Essgewohnheiten!“, „Ernähren Sie sich vollwertig!“ und „Bleiben Sie in Bewegung!"). Auf der linken Innenseite fanden sich zwei weitere Informationseinheiten zu „Adipositas - Was ist das?" und „Was ist der Body-Mass-Index (BMI)?“

Hierin waren alle sechs Versionen identisch. Lediglich die letzten beiden Innenseiten unterschieden sich. Auf diesen Seiten wurde der Flyer in einem 2x3-Design variiert. In 
der einen Version waren die Informationen des Flyers sachlich dargestellt und anhand von statistischen Daten veranschaulicht, während wir die Inhalte in der anderen Version in die Beschreibung eines Fallbeispiels integrierten (vgl. Schaubild A1 im Anhang). Zusätzlich variierten wir das im Flyer enthaltene Bild. ${ }^{2}$ Ein dem Thema angemessenes Bild zu finden, das in drei Stufen variierbar ist, stellte sich als relativ kompliziert heraus. Schließlich sollten sich die Bilder hinsichtlich des Furchtappells deutlich unterscheiden, zugleich jedoch dieselbe Körperregion darstellen, um vergleichbar und ethisch vertretbar zu sein, ohne bestimmte Personen damit zu diskriminieren. Nach mehreren Pretests entschieden wir uns für den Bauch einer weiblichen Person: Einmal war ein sehr dicker Bauch mit Speckfalten und Bindegewebsschwäche (sehr negativ) abgebildet, einmal ein dicker, aber straffer Bauch (leicht negativ) und einmal ein dünner Bauch mit weiter Hose als Indikator für einen erfolgreichen Gewichtsverlust (positiv). ${ }^{3}$ Das Bild war jeweils in eine der oben dargestellten Textversionen eingebettet, so dass wir insgesamt sechs verschiedene Flyerversionen erhielten.

Diese sechs Flyerversionen wurden in einer Druckerei auf 120-Gramm-Papier in Farbe gedruckt, um einen professionell und echt wirkenden Flyer zu gewährleisten (externe Validität). Dass dies gelungen ist, zeigen die Bewertungen der Flyer im Pretest sowie im Experiment selbst. So wurde der Flyer generell als verständlich, eher vertrauenswürdig, sachlich, anschaulich, informativ, glaubwürdig, seriös, überzeugend und gut gemacht beurteilt. Etwas schlechter fiel die Bewertung lediglich im Hinblick auf die Merkmale „interessant - uninteressant“ ( $\mathrm{AM}=2,6$ auf einer Skala von 1 bis 5) und „ansprechend - nicht ansprechend“ $(\mathrm{AM}=2,6)$ aus. Neben der generell guten Bewertung des Flyers ist entscheidend, dass die Flyerqualität unabhängig von der jeweiligen Experimentalversion gleich bleibt. Dies konnten wir bestätigen: Es ließen sich keine signifikanten Unterschiede zwischen den Experimentalgruppen im Hinblick auf ihre Flyerbewertung feststellen.

Zu Ablauf und Fragebogen: Die Versuchspersonen lasen zunächst eine der insgesamt sechs Versionen des Flyers. Anschließend mussten sie den Flyer zurückgeben und einen schriftlichen Fragebogen - vorgeblich zur Qualität von Informationsflyern im Gesundheitsbereich - ausfüllen. Dieser enthielt für die Erhebung des Wissens Fragen zur Definition, zu den Folgen und zur Prophylaxe von Adipositas, woraus ein summarischer Gesamtindex errechnet wurde. ${ }^{4}$ Die Risikowahrnehmung wurde durch Abfrage des geschätzten Anteils Übergewichtiger in Deutschland und des Anteils von Menschen, die aufgrund von Übergewicht gesundheitliche Probleme haben, ermittelt. Zudem wurde die Wahrnehmung des allgemeinen Risikos und die persönliche Sorge, an den Folgeerscheinungen von Adipositas zu erkranken, erhoben. Die Herleitung der Wissens- und Risikofragen orientierte sich eng an den im Flyer dargestellten Inhalten, um diese klar aufeinander beziehen zu können. Die Abfrage ernährungs- und gewichtsbezogener Einstellungen basierte auf einer fünfstufigen Likertskala mit zehn Items. Diese wurden der Skala zu Ernährungseinstellungen der „Typologie der Wünsche“ (z. B. Hubert Burda Media 2006) entlehnt, leicht gekürzt und verändert. Aus zeitlichen Gründen konnten

2 Wir orientierten uns an Zillmann \& Gan (1996), die sich in ihrer Studie auf die Manipulation von Furchtappellen in Bildform konzentrierten (vgl. auch Kapitel 2.1). Konkret konstruierten sie aus einem eher harmlosen neutralen Bild ein deutlich bedrohlicheres Bild, das betroffene Körperstellen zeigte, die einen Großteil der Schulter und des Rückens einnahmen.

3 Die Frage, ob die geplante Bildvalenz der Wahrnehmung durch die Rezipienten entspricht, wurde sowohl vor dem Experiment gesondert getestet, als auch im Experiment anhand von Kontrollfragen zur Bildwahrnehmung kontrolliert und konnte bestätigt werden.

4 Details zur Erhebung der einzelnen Merkmale finden sich in Schaubild A2 im Anhang. 
wir die Skala vorher nicht testen, was sich in der Studie auch als problematisch erwies. Wie aufgrund der unterschiedlichen zugrunde liegenden Dimensionen (gewichtsbezogene und ernährungsbezogene Einstellungen) anzunehmen, erwies sich ein Gesamtindex als nicht reliabel (Cronbach's Alpha=0,55). Deshalb wurden faktorenanalytisch vier Dimensionen identifiziert, die 60 Prozent der Gesamtvarianz erklärten (Genussorientierung, Gesundheitsbewusstsein, Gelassenheit, Gewichtsbewusstsein). Diese konnten jedoch für den zweiten Messzeitpunkt (s. u.) nicht repliziert werden, weshalb für Vergleiche der beiden Messzeitpunkte jeweils Einzelitems herangezogen werden mussten (vgl. hierzu auch Schaubild A2 im Anhang). Anders verhielt es sich bei der Skala zur intendierten Verhaltensänderung bzgl. Ernährung und Bewegung. Auch diese basierte auf einer fünfstufigen Likertskala mit insgesamt neun Items, die sich an den im Flyer vorgegebenen Verhaltensempfehlungen orientierten. Diese Items ließen sich zu einem reliablen Gesamtindex (Cronbach's Alpha=0,85) verdichten. Ferner wurden als intervenierende Variablen Soziodemographie, Körpergröße und Gewicht (Body-MassIndex), Involvement (persönliche Betroffenheit basierend auf sechs Items, Cronbach's Alpha=0,86), physische Aktivität, Nutzung von Gesundheitsflyern und emotionale Reaktionen auf den Flyer abgefragt. Die ebenfalls im ersten Fragebogen erfasste Bewertung des Flyers und die Wahrnehmung des Fotos dienten als Treatment-Check.

Zwei Wochen später füllten die Probanden den zweiten Fragebogen aus, der erneut Wissen, Risikowahrnehmung und Einstellungen abfragte (identische Abfrage wie zum ersten Messzeitpunkt) und zusätzlich erhob, wie sich die Probanden in den zwei Wochen seit der Stimuluspräsentation bezüglich Ernährung und Bewegung verhalten hatten (tatsächliches Verhalten). Letzteres basierte auf denselben Items wie das intendierte Verhalten und ließ sich ebenfalls zu einem reliablen Gesamtindex verdichten (Cronbach's Alpha=0,77). Nach Abschluss des zweiten Fragebogens legten die Versuchsleiter das Untersuchungsziel offen, die Ergebnisse konnten nach einigen Wochen online eingesehen werden.

Insgesamt nahmen 185 Versuchspersonen an dem Experiment teil, 176 Versuchspersonen füllten auch den Fragebogen zum zweiten Messzeitpunkt aus. Die Versuchspersonen wurden von 23 studentischen Versuchsleitern (Teilnehmer des Seminars, in dessen Rahmen die Studie durchgeführt wurde) rekrutiert und nach Gewicht geschichtet (normal- und übergewichtige Personen zu gleichen Teilen), da wir vermuteten, dass der Flyer anders wirkt, wenn man selbst vom Thema betroffen ist. ${ }^{5}$ Es stand den Versuchsleitern frei zu entscheiden, wie und wo sie ihre Versuchspersonen rekrutierten, weshalb viele Personen aus dem Verwandtschafts- oder Freundeskreis der Versuchsleiter

5 So ist zu vermuten, dass Gesundheitsflyer nur von denjenigen Menschen gelesen werden, die sich dieses Informationskanals generell bedienen bzw. ihm gegenüber aufgeschlossen sind. Außerdem werden Menschen mit unterschiedlichem Problembezug auch unterschiedlich auf die Flyer reagieren. Bonfadelli \& Friemel (2006) unterscheiden hier je nach Risikoexposition a) Personen, die nicht durch das Problem (hier: Adipositas) betroffen, aber als Bezugsperson involviert sind, b) Personen, die nicht durch das Problem betroffen, aber „At-Risk“ sind (hier: zu Übergewicht neigende bzw. schon übergewichtige Menschen), c) Personen, die betroffen, aber desinteressiert sind (also desinteressierte Adipöse), d) sensibilisierte Personen, die noch nicht handeln (sensibilisierte Adipöse), und e) Personen, die sensibilisiert sind und handeln (sensibilisierte, handelnde Adipöse). Auch Donovan, Egger \& Francas (1999) stellen durch ihre TARPARE-Analyse u. a. die Aspekte „At-Risk“ (gefährdete Personen, die sich in Einschätzung von Risikofaktoren und Nutzen der Risikoreduktion unterscheiden) und „Persuability“ (Beeinflussbarkeit in Abhängigkeit der Einstellung gegenüber dem erwünschten Verhalten) als zentral heraus, um die Wirkung von Interventionsmaßnahmen vorauszusagen. 
teilnahmen. Jeder Versuchsleiter musste acht Probanden rekrutieren, vier davon übergewichtig, vier normal- oder untergewichtig. Eine zufällige Verteilung der Versuchspersonen zu den Gruppen wurde dadurch gewährleistet, dass jedem Versuchsleiter im Vorfeld in einem Zufallsverfahren unterschiedliche Flyerversionen zugeteilt wurden. Hinsichtlich Alter, Geschlecht und Bildung verteilten sich die Probanden nach dieser Zuteilung gleichmäßig auf die Versuchsgruppen (vgl. Tabelle 1). Da sich wie oben erwähnt in Bezug auf die Bewertung der Flyer keine Unterschiede zwischen den Versionen feststellen lassen, kann davon ausgegangen werden, dass beobachtete Unterschiede in den Ergebnissen auf die Variation der Stimuli zurückgehen.

Tabelle 1: Verteilung der Stichprobe

\begin{tabular}{|c|c|c|c|c|c|}
\hline & \multicolumn{2}{|c|}{ Textversion } & \multicolumn{3}{|c|}{ Bildversion } \\
\hline & $\begin{array}{l}\text { Fallbeispiel } \\
(\mathrm{Fb})\end{array}$ & $\begin{array}{l}\text { summarisch } \\
(\mathrm{sR})\end{array}$ & positiv & $\begin{array}{c}\text { leicht } \\
\text { negativ }\end{array}$ & $\begin{array}{c}\text { sehr } \\
\text { negativ }\end{array}$ \\
\hline Alter (AM) & 38,5 & 37,6 & 37,9 & 39,2 & 37,1 \\
\hline \multicolumn{6}{|l|}{ Geschlecht } \\
\hline weiblich (\%) & 52,6 & 53,9 & 54,1 & 46,7 & 58,7 \\
\hline männlich (\%) & 47,4 & 46,1 & 45,9 & 53,3 & 41,3 \\
\hline \multicolumn{6}{|l|}{ Bildung } \\
\hline kein Abitur (\%) & 33,7 & 27,8 & 23,0 & 36,1 & 33,3 \\
\hline Abitur (\%) & 35,8 & 37,8 & 39,3 & 32,8 & 38,1 \\
\hline $\begin{array}{l}\text { Hochschul- } \\
\text { abschluss (\%) }\end{array}$ & 30,5 & 34,4 & 37,7 & 31,1 & 28,6 \\
\hline \multicolumn{6}{|l|}{ Gewichtsklasse } \\
\hline $\begin{array}{l}\text { Unter-/ } \\
\text { Normal } \\
\text { gewicht (\%) }\end{array}$ & 50,5 & 48,9 & 52,5 & 47,5 & 49,2 \\
\hline $\begin{array}{l}\text { Übergewicht/ } \\
\text { Adipositas (\%) }\end{array}$ & 49,5 & 51,1 & 47,5 & 52,5 & 50,8 \\
\hline BMI (AM) & 24,9 & 25,0 & 25,5 & 25,3 & 24,1 \\
\hline
\end{tabular}

Basis: $\mathrm{n}=182-185$

Anmerkung: Signifikanztests: Chi²-Test bei Geschlecht, Bildung, Gewichtsklasse, t-Test bei Alter und BMI (Textversion), einfaktorielle Varianzanalyse bei Alter und BMI (Bildversion)

Für alle Tests gilt: $\mathrm{p}>0,2$; Ausnahme: Verteilung des BMI auf die Bildversion: $\mathrm{p}=0,17$

\section{Ergebnisse}

Zur Beantwortung von Hypothese 1 wurden im ersten Schritt t-Tests für unabhängige Stichproben gerechnet. Wie Tabelle 2 (Textversion) zeigt, war das Fallbeispiel hier im Gegensatz zu den bisherigen Erkenntnissen zum Fallbeispieleffekt nicht effektiver als die abstrakte summarische Realitätsbeschreibung. Die Versuchspersonen, die den Flyer mit Fallbeispielinformation gelesen hatten, unterschieden sich im Hinblick auf ihr adipositasspezifisches Wissen, Risikowahrnehmung, Einstellungen, Verhaltensintention 
und Verhalten nicht von denen, die den Flyer mit summarischer Realitätsbeschreibung gelesen hatten. Tendenziell war der Flyer mit summarischer Realitätsbeschreibung sogar wirksamer als der Fallbeispiel-Flyer. Hypothese 1 muss daher abgelehnt werden.

Tabelle 2: Einfluss von Text-und Bildversion (Haupteffekte)

\begin{tabular}{|c|c|c|c|c|c|c|c|}
\hline & \multicolumn{3}{|c|}{ Textversion } & \multicolumn{4}{|c|}{ Bildversion } \\
\hline & $\begin{array}{c}\mathbf{F b} \\
(\mathrm{n}=92-95)\end{array}$ & $\begin{array}{c}\mathbf{s R} \\
(\mathrm{n}=86-89)\end{array}$ & $\mathrm{t}$ & $\begin{array}{c}\text { positiv } \\
(n=56-61)\end{array}$ & $\begin{array}{c}\text { leicht } \\
\text { negativ } \\
(n=59-61)\end{array}$ & $\begin{array}{c}\text { sehr } \\
\text { negativ } \\
(\mathrm{n}=60- \\
63)\end{array}$ & F \\
\hline Wissen & 5,8 & 6,1 & 1,57 & $6,1^{a}$ & $5,5^{\mathrm{b}}$ & $6,3^{a}$ & $3,75^{*}$ \\
\hline Risiko & & & & & & & \\
\hline $\begin{array}{l}\text { Risiko zu } \\
\text { erkranken }\end{array}$ & 2,8 & 2,8 & 0,09 & 2,7 & 3,0 & 2,8 & 0,61 \\
\hline $\begin{array}{l}\text { Sorge zu } \\
\text { erkranken }\end{array}$ & 1,8 & 1,8 & 0,03 & 1,8 & 1,9 & 1,8 & 0,37 \\
\hline $\begin{array}{l}\text { Anteil } \\
\text { Über- } \\
\text { gewichtiger }\end{array}$ & 48,6 & 49,1 & 0,21 & 47,6 & 49,1 & 49,7 & 0,36 \\
\hline $\begin{array}{l}\text { Anteil } \\
\text { Kranker }\end{array}$ & 39,4 & 43,4 & 1,38 & $37,6^{\mathrm{a}}$ & $47,2^{\mathrm{b}}$ & $39,2^{a}$ & $4,22 *$ \\
\hline Einstellung & & & & & & & \\
\hline Genuss & 0,1 & $-0,1$ & 0,89 & 0,1 & 0,0 & $-0,1$ & 1,04 \\
\hline Gesundheit & $-0,0$ & 0,0 & 0,84 & $-0,2$ & 0,2 & 0,0 & 2,31 \\
\hline Gelassenheit & 0,1 & $-0,1$ & 0,38 & $-0,0$ & $-0,0$ & 0,0 & 0,06 \\
\hline Gewicht & 0,0 & $-0,0$ & 0,55 & $-0,1$ & 0,1 & 0,0 & 0,28 \\
\hline $\begin{array}{l}\text { Verhaltens- } \\
\text { intention }\end{array}$ & 2,3 & 2,5 & 1,33 & 2,4 & 2,5 & 2,4 & 0,35 \\
\hline Verhalten & 2,2 & 2,2 & 0,57 & $2,0^{a}$ & $2,3^{\mathrm{b}}$ & $2,3^{\mathrm{b}}$ & $3,20 *$ \\
\hline
\end{tabular}

Basis: $\mathrm{n}=176-184$, t-Test bzw. einfaktorielle Varianzanalyse, Mittelwerte mit unterschiedlichen Kennbuchstaben unterscheiden sich signifikant nach dem Duncan-Test für Mittelwertunterschiede bei unabhängigen Stichproben, ${ }^{*} \mathrm{p}<0,05, * \mathrm{p}<0,01$

Etwas anders stellen sich die Unterschiede dar, wenn man das Involvement in die Berechnung miteinbezieht. Während Alter, Bildung, eigenes Körpergewicht (BMI), physische Aktivität und die generelle Nutzung von Gesundheitsflyern den Einfluss des Fallbeispiels nicht determinieren (wie in Hypothese 2 postuliert, gab es hier keine Interaktionseffekte), lassen sich unter Berücksichtigung des Involvements zumindest teilweise Unterschiede feststellen (vgl. Tabelle 3): Erwartungsgemäß schätzten die hoch involvierten Probanden das eigene und generelle Risiko, an Adipositas zu erkranken, höher ein als niedrig Involvierte. Zudem zeigten Erstere ernährungsbewusstere Einstellungen und Verhaltensintentionen und gaben nach zwei Wochen an, sich besser ernährt und mehr bewegt zu haben (Verhalten). In Bezug auf adipositasspezifisches Wissen und 
Gewichtsbewusstsein fiel der Effekt noch stärker aus, wenn die Probanden den Flyer mit Fallbeispiel gelesen hatten. Bei hoch involvierten Personen zeigte sich also, auf niedrigem Niveau, der vermutete Fallbeispieleffekt, der sich in der Gesamtstichprobe nicht beobachten ließ. Dieses Muster findet sich zwar tendenziell bei den meisten anderen Merkmalen wieder, jedoch sind die Unterschiede meist nicht signifikant und nur sehr klein, weshalb der interagierende Einfluss des Involvement insgesamt als gering eingeschätzt werden muss.

Tabelle 3:

Interaktion zwischen Textversion und Involvement

\begin{tabular}{|c|c|c|c|c|c|c|c|c|c|}
\hline & \multicolumn{2}{|c|}{$\mathrm{Fb}$} & \multicolumn{2}{|c|}{$s R$} & \multirow{2}{*}{$\frac{\text { Text }}{\mathrm{F}}$} & \multirow{2}{*}{$\frac{\text { Involv. }}{\mathrm{F}}$} & \multirow{2}{*}{$\frac{\mathrm{T}^{*} \mathrm{I}}{\mathrm{F}}$} & \multicolumn{2}{|c|}{ Gesamt } \\
\hline & $\begin{array}{c}\text { Involv. } \\
\text { niedrig } \\
(\mathrm{n}=53- \\
55)\end{array}$ & $\begin{array}{c}\text { Involv. } \\
\text { hoch } \\
(\mathrm{n}=35- \\
40)\end{array}$ & $\begin{array}{c}\text { Involv. } \\
\text { niedrig } \\
(\mathrm{n}=41- \\
44)\end{array}$ & $\begin{array}{c}\text { Involv. } \\
\text { hoch } \\
(\mathrm{n}=43- \\
45)\end{array}$ & & & & F & $\begin{array}{c}\mathrm{R}^{2} \\
\text { (korr.) }\end{array}$ \\
\hline Wissen & 5,4 & 6,2 & 6,4 & 5,9 & 6,71 & 0,15 & $6,71 \%$ & $3,14 *$ & 0,03 \\
\hline \multicolumn{10}{|l|}{ Risiko } \\
\hline $\begin{array}{l}\text { Risiko zu } \\
\text { erkranken }\end{array}$ & 2,5 & 3,4 & 2,4 & 3,3 & 0,10 & $21,17 * *$ & 0,00 & $7,07 * *$ & 0,10 \\
\hline $\begin{array}{l}\text { Sorge zu } \\
\text { erkranken }\end{array}$ & 1,4 & 2,3 & 1,4 & 2,2 & 0,30 & $41,42 * *$ & 0,04 & $13,82 * *$ & 0,18 \\
\hline $\begin{array}{l}\text { Anteil } \\
\text { Über- } \\
\text { gewichtig. }\end{array}$ & 47,9 & 50,9 & 48,6 & 49,5 & 0,02 & 0,82 & 0,23 & 0,35 & $-0,01$ \\
\hline $\begin{array}{l}\text { Anteil } \\
\text { Kranker }\end{array}$ & 36,4 & 43,7 & 42,2 & 44,2 & 1,04 & 2,34 & 0,76 & 1,57 & 0,01 \\
\hline \multicolumn{10}{|l|}{ Einstellung } \\
\hline Genuss & 0,1 & 0,0 & $-0,1$ & 0,0 & 0,52 & 0,06 & 0,46 & 0,36 & $-0,01$ \\
\hline Gesundheit & $-0,2$ & 0,2 & $-0,1$ & 0,1 & 0,02 & $4,02 *$ & 0,10 & 1,38 & 0,01 \\
\hline $\begin{array}{l}\text { Gelassen- } \\
\text { heit }\end{array}$ & $-0,1$ & 0,3 & $-0,1$ & $-0,1$ & 1,64 & 2,51 & 1,29 & 1,66 & 0,01 \\
\hline Gewicht & $-0,5$ & 0,8 & $-0,4$ & 0,3 & 1,78 & $54,44 \%$ & $4,45^{*}$ & $19,79 * *$ & 0,24 \\
\hline $\begin{array}{l}\text { Verhaltens- } \\
\text { intention }\end{array}$ & 1,8 & 3,0 & 2,2 & 2,9 & 0,75 & $47,83 \%$ & 3,47 & $18,32 * *$ & 0,23 \\
\hline Verhalten & 2,0 & 2,4 & 2,0 & 2,4 & 0,00 & $18,65 * \%$ & 0,05 & $6,31 * \%$ & 0,08 \\
\hline
\end{tabular}

Basis: $\mathrm{n}=174-183$, zweifaktorielle Varianzanalyse, ${ }^{*} \mathrm{p}<0,05, * \mathrm{p}<0,01$

Während im Zusammenhang mit der Textversion also keine Gruppenunterschiede festgestellt werden konnten, lassen sich im Zusammenhang mit der Bildvalenz (Forschungsfrage 1) zumindest teilweise signifikante Unterschiede beobachten (vgl. Tabelle 2, Bildversion). So zeigten sich beim Wissen Unterschiede, die eine U-Funktion nachzeichnen, d. h. Probanden, die den Flyer mit positivem und sehr negativem Bild gelesen hatten, wussten hinterher mehr als die dritte Gruppe mit dem leicht negativen Bild. Beim Verhalten war die Wirkung des leicht negativen und des negativen Bildes am stärksten, und 
bei einer von vier Risikowahrnehmungsdimensionen zeigten sich signifikante Unterschiede, die eine umgekehrte U-Funktion nachzeichnen: Das leicht negative Bild hatte hier also einen signifikant stärkeren Einfluss auf die Risikowahrnehmung als das positive und sehr negative Bild. Diese umgekehrte U-Funktion ließ sich zumindest tendenziell auch bei anderen Merkmalen beobachten, konkret beim wahrgenommenen Risiko und der Sorge zu erkranken, bei gesundheits- und gewichtsbezogenen Einstellungen und bei den Verhaltensintentionen. Damit entspricht der hier beobachtete Einfluss der Bildvalenz zumindest in Teilen den bisherigen Erkenntnissen zum Furchtappell bzw. zur Wirkung von Bildern.

Die divergierende Wirkung von Bildern unterschiedlicher Valenz wird häufig darauf zurückgeführt, dass sie unterschiedliche emotionale Reaktionen hervorrufen (vgl. z. B. Keller 2008; Forschungsfrage 2). Diese Vermutung wurde durch die Analyse von Interaktionseffekten geprüft, konnte allerdings nicht bestätigt werden. Weder die allgemeine Stärke der emotionalen Reaktion (Gesamtindex), noch Mitleid oder Betroffenheit (Einzeldimensionen) veränderten die beobachteten Gruppenunterschiede. Der Einfluss der restlichen intervenierenden Merkmale (Soziodemographie, Flyernutzung, physische Aktivität, BMI, Involvement) entspricht im Großen und Ganzen den Befunden zum Fallbeispieleffekt. So lassen sich nur beim Involvement geringfügige Interaktionseffekte feststellen, die jedoch meist nicht signifikant sind (vgl. Tabelle 4).

Tabelle 4: Interaktion zwischen Bildvalenz und Involvement

\begin{tabular}{|c|c|c|c|c|c|c|c|c|c|c|c|}
\hline & \multicolumn{2}{|c|}{ positiv } & \multicolumn{2}{|c|}{ leicht negativ } & \multicolumn{2}{|c|}{ sehr negativ } & \multirow{2}{*}{\begin{tabular}{|c|} 
Bild \\
F
\end{tabular}} & \multirow{2}{*}{$\frac{\text { Involv. }}{\mathrm{F}}$} & \multirow{2}{*}{$\frac{\mathrm{B}^{*} \mathrm{I}}{\mathrm{F}}$} & \multicolumn{2}{|c|}{ Gesamt } \\
\hline & $\begin{array}{c}\text { Involv. } \\
\text { niedrig } \\
(\mathrm{n}= \\
29-31)\end{array}$ & $\begin{array}{c}\text { Involv. } \\
\text { hoch } \\
(\mathrm{n}= \\
27-29)\end{array}$ & $\begin{array}{c}\text { Involv. } \\
\text { niedrig } \\
(\mathrm{n}= \\
31-32)\end{array}$ & $\begin{array}{c}\text { Involv. } \\
\text { hoch } \\
(\mathrm{n}= \\
27-29)\end{array}$ & $\begin{array}{c}\text { Involv. } \\
\text { niedrig } \\
(\mathrm{n}= \\
35-37)\end{array}$ & $\begin{array}{l}\text { Involv. } \\
\text { hoch } \\
(\mathrm{n}= \\
23-26)\end{array}$ & & & & $\mathrm{F}$ & $\begin{array}{c}\mathrm{R}^{2} \\
\text { (korr.) }\end{array}$ \\
\hline Wissen & 5,8 & 6,3 & 5,1 & 5,9 & 6,6 & 5,8 & $3,21 *$ & 0,54 & $3,76 *$ & $3,14 *$ & 0,06 \\
\hline \multicolumn{12}{|l|}{ Risiko } \\
\hline $\begin{array}{l}\text { Risiko zu } \\
\text { erkranken }\end{array}$ & 2,3 & 3,2 & 2,6 & 3,4 & 2,5 & 3,4 & 0,78 & $21,55^{* *}$ & 0,07 & $4,55 \%$ & 0,09 \\
\hline $\begin{array}{l}\text { Sorge zu } \\
\text { erkranken }\end{array}$ & 1,4 & 2,1 & 1,4 & 2,4 & 1,4 & 2,4 & 0,53 & $41,53 \%$ & 0,92 & $8,80 * *$ & 0,18 \\
\hline $\begin{array}{l}\text { Anteil } \\
\text { Übergew. }\end{array}$ & 46,6 & 49,8 & 46,2 & 52,0 & 51,1 & 48,2 & 0,16 & 0,89 & 1,45 & 0,86 & $-0,00$ \\
\hline $\begin{array}{l}\text { Anteil } \\
\text { Kranker }\end{array}$ & 34,7 & 41,8 & 40,9 & 52,8 & 40,8 & 36,3 & $3,64 *$ & 2,62 & 2,66 & $2,88^{*}$ & 0,05 \\
\hline \multicolumn{12}{|l|}{ Einstellung } \\
\hline Genuss & 0,1 & 0,2 & 0,0 & 0,0 & $-0,1$ & $-0,2$ & 1,03 & 0,01 & 0,04 & 0,42 & $-0,02$ \\
\hline Gesund. & $-0,4$ & $-0,0$ & 0,1 & 0,3 & $-0,1$ & 0,2 & 2,40 & $4,28^{*}$ & 0,03 & 1,79 & 0,02 \\
\hline $\begin{array}{l}\text { Gelassen- } \\
\text { heit }\end{array}$ & $-0,1$ & 0,1 & $-0,1$ & 0,0 & $-0,1$ & 0,2 & 0,10 & 2,25 & 0,12 & 0,52 & $-0,01$ \\
\hline Gewicht & $-0,4$ & 0,3 & $-0,5$ & 0,6 & $-0,5$ & 0,7 & 0,57 & $52,60 * \%$ & 1,13 & $11,16^{* *}$ & 0,22 \\
\hline $\begin{array}{l}\text { Verhaltens- } \\
\text { intention }\end{array}$ & 2,0 & 2,9 & 2,0 & 3,1 & 2,0 & 2,9 & 0,18 & $48,24 * *$ & 0,33 & $9,97 * *$ & 0,21 \\
\hline Verhalten & 2,0 & 2,1 & 2,0 & 2,6 & 2,0 & 2,6 & $4,14 *$ & $20,40 \cdots \cdots$ & 2,58 & $6,56^{* *}$ & 0,14 \\
\hline
\end{tabular}

Basis: $\mathrm{n}=174-182$, zweifaktorielle Varianzanalyse, ${ }^{*} \mathrm{p}<0,05, * \mathrm{p}<0,01$ 
Die beobachtete umgekehrte U-Funktion bleibt bei hoch involvierten Personen erhalten, wenn es um die Einschätzung des Anteils Übergewichtiger und Kranker geht, um Einstellungen zur Gesundheit oder um Verhaltensintentionen. Ansonsten verschwindet sie weitgehend und der Gesundheitsflyer erzielt mit den negativen Bildern tendenziell eine stärkere Wirkung. Das leicht oder sehr negative Bild ist dann bei hoch involvierten Personen tendenziell wirkungsvoller als das positive. Hier lässt sich das zumindest für die Einschätzung des Risikos und der Sorge zu erkranken, für gewichtsspezifische Einstellungen und Verhalten bestätigen. Diese Tendenz würde den Ausführungen von Job (1988) entsprechen, wonach Furchtappelle am besten wirken, wenn sich die Zielgruppe schon vorher von einem Problem betroffen fühlt, also involviert ist. Unsere Beobachtungen basieren jedoch auf Tendenzen und sind daher mit Vorsicht zu betrachten. Der einzige signifikante Unterschied ließ sich beim Wissen feststellen und hier kehrt sich das Muster um: Der Flyer wirkt umso besser, je positiver das Bild ist, d. h. hoch involvierte Personen lernen vom Flyer mit positivem Bild am meisten.

Im nächsten Schritt ging es um die Frage, ob sich auch Interaktionseffekte zwischen den beiden Faktoren, also zwischen Fallbeispiel und Bildvalenz, beobachten lassen (Forschungsfrage 3). Erneut wurden mehrfaktorielle Varianzanalysen gerechnet. Nur beim Wissen konnte jedoch ein signifikanter Interaktionseffekt nachgewiesen werden, wonach sich der Einfluss der Bildvalenz (U-Funktion) in der Fallbeispielversion eher zeigt, zusammen mit der summarischen Realitätsbeschreibung aber fast verschwindet. ${ }^{6}$ Insgesamt wussten die Probanden, die den Flyer mit sehr negativem Bild und summarischer Realitätsbeschreibung gelesen hatten, hinterher am meisten. Damit unterschieden sie sich am deutlichsten von denen, die die Fallbeispielversion mit leicht negativem Bild gelesen hatten.

Zuletzt galt es herauszufinden, wie stabil die beobachteten Effekte sind (Forschungsfrage 4, Tabelle 5). Hierfür wurden Wissen, Risikowahrnehmung und Einstellungen zwei Wochen nach der Stimuluspräsentation erneut gemessen. Im ersten Schritt rechneten wir jeweils gepaarte t-Tests zwischen den erfassten Merkmalen des ersten und zweiten Messzeitpunktes. Nur bei einzelnen Merkmalen zeigten sich signifikante Veränderungen zwischen den Messzeitpunkten. So ließ die Wirkung auf ernährungsbezogene Einstellungen nach zwei Wochen zum Teil signifikant nach. Zudem lässt sich erkennen, dass die Probanden nach zwei Wochen - zwar nicht signifikant, aber in der Tendenz weniger wussten und das Risiko, an Adipositas zu erkranken, geringer einschätzten. Trotz dieser leichten Differenzen deutet dies darauf hin, dass die Intervention nicht vollkommen an den Probanden vorbeiging. Zumindest für den Zeitraum von 14 Tagen scheinen die Effekte relativ stabil zu sein.

Diese Unterschiede sind unabhängig von Textversion und Bildvalenz. Um dies festzustellen, berechneten wir zunächst jeweils die Differenz zwischen den zum ersten und zweiten Messzeitpunkt erfassten Merkmalen und bildeten daraus neue Variablen. Mit t-Tests (Textversion) bzw. einfaktoriellen Varianzanalysen (Bildversion), in die die neu errechneten Differenz-Variablen als abhängige Variablen eingingen, wurde dann der Einfluss der beiden Faktoren auf die Stabilität der Einflüsse gemessen. Wie Tabelle 5 zeigt, gibt es keine systematischen Unterschiede zwischen den Messzeitpunkten und der Text- bzw. Bildversion.

6 Konkret lagen die arithmetischen Mittel in der Fallbeispielversion bei 6,1 (positives Bild), 5,0 (leicht negativ) und 6,1 (sehr negativ), hingegen in der summarischen Realitätsbeschreibung bei 6,0 (positiv), 5,9 (leicht negativ) und 6,5 (sehr negativ) ( $\mathrm{F}$ (Text*Bild-Interaktion) $=2,51$, $\mathrm{p}<0,05)$. 
Tabelle 5: Stabilität der Effekte

\begin{tabular}{|c|c|c|c|c|c|c|c|c|c|c|c|}
\hline & \multicolumn{4}{|c|}{ Gesamt } & \multicolumn{3}{|c|}{$\begin{array}{l}\text { Textversion } \\
\text { (Differenz) }\end{array}$} & \multicolumn{4}{|c|}{$\begin{array}{l}\text { Bildversion } \\
\text { (Differenz) }\end{array}$} \\
\hline & $\mathrm{AM} \mathrm{t}_{1}$ & $\mathrm{AM} \mathrm{t}_{2}$ & $\mathrm{AM}_{\text {dif }}$ & $\mathrm{t}$ & $\mathrm{Fb}$ & $s R$ & $\mathrm{t}$ & pos. & 1. neg. & s. neg. & $\mathrm{F}$ \\
\hline Wissen & 5,95 & 5,87 & $-0,08$ & 0,69 & 0,12 & $-0,31$ & 1,80 & $-0,02$ & $-0,07$ & $-0,15$ & 0,11 \\
\hline \multicolumn{12}{|l|}{ Risiko } \\
\hline $\begin{array}{l}\text { Risiko zu } \\
\text { erkranken }\end{array}$ & 2,83 & 2,49 & $-0,33$ & 3,26 & $-0,30$ & $-0,36$ & 0,29 & $-0,01$ & $-0,44$ & $-0,52$ & 2,40 \\
\hline $\begin{array}{l}\text { Sorge zu } \\
\text { erkranken }\end{array}$ & 1,82 & 1,77 & $-0,05$ & 1,02 & 0,03 & $-0,13$ & 1,65 & $0,13^{\mathrm{a}}$ & $-0,17^{b}$ & $-0,09^{\mathrm{ab}}$ & $3,81 \%$ \\
\hline $\begin{array}{l}\text { Anteil } \\
\text { Übergew. }\end{array}$ & 48,60 & 48,66 & 0,07 & 0,08 & 0,89 & $-0,78$ & 0,98 & 1,61 & $-1,82$ & 0,48 & 1,42 \\
\hline $\begin{array}{l}\text { Anteil } \\
\text { Kranker }\end{array}$ & 41,04 & 40,39 & $-0,66$ & 0,56 & $-0,02$ & $-1,32$ & 0,56 & 0,18 & $-1,90$ & $-0,20$ & 0,30 \\
\hline \multicolumn{12}{|l|}{ Einstellung ${ }^{1}$} \\
\hline Genuss & 3,85 & 3,71 & $-0,13$ & $2,15^{*}$ & $-0,11$ & $-0,15$ & 0,34 & $-0,14$ & $-0,18$ & $-0,07$ & 0,31 \\
\hline Gesund. & 4,29 & 4,39 & 0,10 & 1,79 & 0,12 & 0,08 & 0,36 & 0,25 & 0,03 & 0,03 & 1,58 \\
\hline Gelassenheit & 2,76 & 2,91 & 0,15 & 1,80 & 0,10 & 0,21 & 0,64 & 0,27 & 0,18 & 0,02 & 0,74 \\
\hline Gewicht & 2,93 & 2,75 & $-0,18$ & $2,50 *$ & $-0,29$ & $-0,05$ & 1,48 & $-0,05$ & $-0,27$ & $-0,18$ & 0,55 \\
\hline
\end{tabular}

Basis: $\mathrm{n}($ gesamt $)=169-176, \mathrm{n}($ Fallbeispiel $)=87-90, \mathrm{n}($ summarisch $)=82-86, \mathrm{n}($ positiv $)=53-56, \mathrm{n}$ (leicht negativ) $=57-60, \mathrm{n}$ (sehr negativ) $=58-60$, gepaarte $\mathrm{t}$-Tests, $\mathrm{t}$-Test für unabhängige Stichproben bzw. einfaktorielle Varianzanalyse, Mittelwerte mit unterschiedlichen Kennbuchstaben unterscheiden sich signifikant nach dem Duncan-Test für Mittelwertunterschiede bei unabhängigen Stichproben, $* \mathrm{p}<0,05, * \mathrm{p}<0,01$

${ }^{1}$ Anmerkung: Da sich aus den zum zweiten Messzeitpunkt erhobenen Einstellungsitems nicht dieselben Faktoren extrahieren ließen wie zum ersten Messzeitpunkt, wurde für diese Auswertung jeweils nur ein Item stellvertretend für die Einstellungsdimension herangezogen, und zwar jeweils das Item, welches bei der Faktorenanalyse der Einstellungsitems des ersten Messzeitpunktes am stärksten auf den jeweiligen Dimensionen lud. Konkret waren dies die folgenden Items: Genuss „Essen ist für mich eines der größten Vergnügen.“, Gesundheit - „Es ist wichtig, auf eine gesunde, ausgewogene Ernährung zu achten.“, Gelassenheit - „Attraktivität hat nichts mit Körpergewicht zu tun.“, Gewichtsbewusstsein - „Ich bin oft neidisch, wenn ich jemanden mit einer guten Figur sehe."

\section{Zusammenfassung und Diskussion}

Zusammenfassend kann man festhalten, dass die Verwendung eines Fallbeispiels im Vergleich zur Darstellung in Form einer abstrakten summarischen Realitätsbeschreibung in Gesundheitsflyern nach diesen Befunden nicht effektiver ist. Bei keiner der untersuchten abhängigen Variablen (Wissen, Risikowahrnehmung, Einstellungen, Handlungsintention, Verhalten) waren Unterschiede durch die Textversion zu beobachten. Dies bedeutet jedoch nicht zwangsläufig, dass Fallbeispiele im Rahmen von Gesundheitsflyern unwirksam sind. Möglicherweise lassen sich die beobachteten Befunde damit erklären, dass wir nur ein Fallbeispiel verwendet haben. Das widerspricht dem Setting klassischer Fallbeispielstudien. Diese präsentieren in der Regel mehrere Fallbeispiele - häufig auch Fallbeispiele, die für jeweils unterschiedliche Aussagen stehen - und stellen sie 
einer summarischen Realitätsbeschreibung gegenüber. Meist errechnen die Probanden etwa die Einschätzung eines bestimmten Risikos oder des Meinungsklimas zu einem bestimmten Thema dann direkt aus der Anzahl der (betroffenen vs. nicht betroffenen oder befürwortenden vs. gegnerischen) Fallbeispiele (vgl. z. B. Zillmann, Perkins \& Sundar 1992). Dieses Setting ist auf die Verwendung von Fallbeispielen in Gesundheitsflyern leicht zu übertragen: Anstatt nur einen Betroffenen (hier: eine übergewichtige Person) zu beschreiben, würde man mehrere Betroffene darstellen, evtl. auch Personen, die an einem Problem nicht mehr leiden oder gar nie darunter gelitten haben. Damit könnte man verdeutlichen, dass es möglich ist, durch eigenes Verhalten bzw. Verhaltensänderungen gesund zu werden oder zu bleiben. Gleichzeitig ließe sich damit auch eine realistischere Risikoverteilung abbilden. Dies müsste in Folgestudien noch genauer geklärt werden.

Im Gegensatz zum Einfluss des Fallbeispiels lassen sich im Zusammenhang mit der Bildversion zumindest tendenziell unterschiedliche Befunde festhalten. Bei Risikowahrnehmung, Einstellungen und Verhaltensintentionen lässt sich in weiten Teilen durch das leicht negative Bild die beste Wirkung erzielen, während das sehr negative und das positive Bild ähnlich schlecht abschneiden. Beim Wissen ist es genau umgekehrt, $\mathrm{d}$. h. hier erzielten die Versionen mit dem positiven und dem sehr negativen Bild die beste Wirkung. Möglicherweise lässt sich der Unterschied damit erklären, dass sowohl das positive als auch das sehr negative Bild auffälliger sind, wodurch sie vielleicht mehr Aufmerksamkeit erzeugen. Zillmann, Knobloch \& Yu (2001) stützen diese Interpretation zumindest für das negative Bild. Denn die Befunde ihrer Studie deuten darauf hin, dass dramatisierende Bilder die Aufmerksamkeit des Lesers wecken, ihn länger bei einem Artikel verweilen und diesen mit größerer Aufmerksamkeit lesen lassen. Aufmerksamkeit allein reicht jedoch offenbar nicht aus, um auch Risikowahrnehmung, Einstellungen und Verhaltensintentionen zu beeinflussen. Bei diesen Merkmalen wirkt das leicht negative Bild im Gegensatz zum positiven vermutlich deshalb besser, weil es eine noch stärkere Appellfunktion hat, und im Vergleich zum sehr negativen Bild, weil es dabei keine Ablehnung und Furcht erzeugt. Lediglich für eine Veränderung des tatsächlichen Verhaltens ist nach unseren Befunden auch das Bild mit starkem Furchtappell geeignet, da hier das leicht negative und sehr negative Bild in ihrer Wirkung gleichauf waren und zu einer stärkeren Verhaltensänderung führten als das positive.

Bezieht man das Involvement wieder mit in die Betrachtung ein, sehen die Unterschiede etwas anders aus: Die beobachtete umgekehrte U-Funktion bleibt bei hoch involvierten Personen erhalten, wenn es um die Einschätzung der Anteile Betroffener geht, um Einstellungen zu Gesundheit oder Verhaltensintentionen. Ansonsten verschwindet sie weitgehend und der Gesundheitsflyer erzielt mit den negativen Bildern tendenziell ein stärkere Wirkung. So waren bei der Einschätzung des Risikos und der Sorge zu erkranken, bei gewichtsspezifischen Einstellungen und Verhalten die beiden negativen Bilder in der Gruppe der hoch involvierten Personen tendenziell wirkungsvoller als das positive. Diese Tendenz würde den Ausführungen von Job (1988) entsprechen, wonach Furchtappelle am besten wirken, wenn sich die Zielgruppe schon vorher von einem Problem betroffen fühlt, also involviert ist. Allerdings basieren diese Beobachtungen auf nicht signifikanten Unterschieden. Der einzige signifikante Unterschied ließ sich beim Wissen feststellen und das Muster kehrt sich um: Hier wirkt der Flyer also umso besser, je positiver das Bild ist, d. h. hoch involvierte Personen lernen vom Flyer mit positivem Bild am meisten.

Die beobachteten Ergebnisse sind jedoch mit Einschränkung zu betrachten. Das Hauptproblem der Studie wurde bereits angesprochen: So beruht die Fallbeispielvaria- 
tion lediglich auf der Verwendung eines Fallbeispiels, während Fallbeispieleffekte meist dann beobachtet werden, wenn mehrere Fallbeispiele vorhanden sind. Dies könnte erklären, weshalb wir keine Fallbeispieleffekte gefunden haben. Denkbar ist aber auch, dass der Anteil variierter Textpassagen zu gering war, um Fallbeispieleffekte beobachten zu können. So enthielt auch die Fallbeispielversion Textpassagen, die die Sachverhalte ähnlich einer summarischen Realitätsbeschreibung darstellten (vgl. z. B. den dritten Abschnitt in Schaubild A1, Anhang). Wir entschieden uns aus Gründen der externen Validität dafür, nicht alle Textpassagen zu variieren, zumal wir aus Fallbeispielstudien wissen, dass Fallbeispielinformationen trotz ebenfalls rezipierter summarischer Realitätsinformation stärker wirken. Dennoch ist denkbar, dass dies, neben der Tatsache, dass nur ein Fallbeispiel verwendet wurde, dafür mitverantwortlich ist, dass wir keine Unterschiede feststellen konnten. Dies sollte in zukünftigen Studien aufgegriffen werden, um klare Aussagen über die Funktion von Fallbeispielen im Kontext von Gesundheitsinformationen treffen zu können.

Auch im Hinblick auf den zweiten Faktor des Experiments - die Variation der Bilder - ist zu überlegen, ob sich hier nicht klarere Unterschiede finden ließen, wenn man die Art der Operationalisierung ändern würde. Wir entschieden uns dazu, den Furchtappell beim Thema Adipositas in Form von unterschiedlichen Bauchausschnitten (schlank und hübsch vs. dick mit leicht negativer Anmutung vs. sehr dick und mit negativer Anmutung) darzustellen, um das inhaltlich Gezeigte möglichst konstant zu halten und somit nur den Grad des Furchtappells zu variieren. Im Treatment-Check zeigte sich durchaus, dass die unterschiedlich anmutenden Bilder entsprechend unserer Variation wahrgenommen und bewertet wurden. Allerdings stellt sich vor dem Hintergrund der in der Furchtappellforschung üblichen Verwendung von angegriffenen (und häufig unansehnlich entstellten) Organen oder Körperteilen die Frage, ob die Operationalisierung des starken Furchtappells in dieser Studie tatsächlich vergleichbar ist mit anderen möglicherweise deutlich furchterregenden Formen des Furchtappells.

Ein weiteres Problem betrifft den Zeitpunkt der Studie: Konzeptioniert und durchgeführt wurde sie im Rahmen eines Forschungsseminars im Wintersemester. Die Datenerhebung fiel somit genau auf die Weihnachtszeit. Vor diesem Hintergrund ist es denkbar, dass die Messung zum zweiten Messzeitpunkt verzerrt ist, da sich die Probanden in der Zeit nach Weihnachten entweder besonders stark mit dem Thema Essen oder Diät beschäftigt haben oder besonders wenig. Geht man allerdings davon aus, dass dies auf alle Probanden zutrifft bzw. sich aufgrund der Zufallsauswahl die möglichen unterschiedlichen Effekte der Weihnachtszeit gleichmäßig über die Gruppen verteilen, kann das in Kauf genommen werden. Eine Wiederholungsmessung zu einem anderen Zeitpunkt würde dennoch mehr Sicherheit geben.

Eine weitere Einschränkung betrifft die Auswahl des Themas. Es ist denkbar, dass die insgesamt geringen und nur teilweise signifikanten Gruppenunterschiede auf einen Deckeneffekt zurückgehen, da die Bevölkerung grundsätzlich bereits gut über Ursachen und Risiken von Adipositas und Übergewicht Bescheid weiß und auch Einstellungen und Verhaltensintentionen insgesamt nur wenig Varianz aufweisen. Es könnte daher sein, dass sich bei anderen, weniger bekannten Krankheitsbildern oder Gesundheitsrisiken deutlichere Unterschiede zeigen. Dies gilt es jedoch erst zu prüfen. Auch die Messung ernährungs- und gewichtsbezogener Einstellungen sollte in zukünftigen Studien erneut überprüft werden. Die von uns aus verschiedenen Studien entwickelte Skala war nicht geeignet, die Einstellungen in einem Index abzubilden (mangelnde Reliabilität). Auf der Basis von Faktorenanalysen konnten vier Dimensionen extrahiert werden, die sich jedoch bei den identisch abgefragten Einstellungen des zweiten Mess- 
zeitpunktes nicht replizieren ließen. Die einstellungsbezogenen Befunde müssen daher mit Einschränkung betrachtet werden und bedürfen ebenfalls weiterer Überprüfung.

Ein letztes Problem betrifft schließlich die Tatsache, dass wir in unserer Untersuchung keine Vorhermessung durchgeführt haben. Wir können also nichts darüber aussagen, was die Probanden über Adipositas wussten, wie sie die Risiken wahrgenommen haben, welche Einstellungen zu Ernährung und Bewegung sie hatten und wie ihre Ernährungsgewohnheiten waren, bevor sie den Flyer lesen mussten. Wir verzichteten bewusst darauf, die abhängigen Variablen vor der Flyerrezeption zu messen, um zu verhindern, dass den Probanden zu früh klar wird, worum es geht. Vermutlich hätte dies die Flyerrezeption zu stark verzerrt. Durch die bis auf die manipulierten Faktoren strukturgleichen Gruppen dürfte aber gewährleistet sein, dass die beobachteten Unterschiede auf die Manipulation zurückgehen und nicht auf etwaige Unterschiede in den Voreinstellungen.

Sieht man von den dargestellten Einschränkungen ab, lassen sich folgende Empfehlungen für die Gestaltung von Flyern festhalten: Während Fallbeispiele nach unseren Befunden für die Wirksamkeit von Gesundheitsflyern nur eine geringe Bedeutung spielen, lassen sich durch den bewussten Einsatz bestimmter Bilder durchaus bessere Ergebnisse erzielen. Wie aus der Literatur zur Gesundheitsprävention hinlänglich bekannt (Donovan 2007, Hurrelmann, Klotz \& Haisch 2007) spielt auch hier die Zielgruppe eine wichtige Rolle. So hängt die Auswahl des richtigen Bildes stark davon ab, welche Zielgruppe und welches Ziel erreicht werden soll. Es macht einen Unterschied, ob man Personen ansprechen will, die bereits involviert sind, oder Personen, bei denen man Interesse und Involvement erst wecken muss: Dient der Flyer hauptsächlich dazu, dass die Rezipienten mehr über ein Thema erfahren (Wissen), ist es ratsam, eher positive oder sehr negative Bilder zu wählen. Will man die Rezipienten hingegen dazu bringen, Einstellungen oder Verhaltensintentionen zu ändern, ist es vermutlich ratsamer, leicht negative Bilder zu verwenden. Für eine tatsächliche Verhaltensänderung eignen sich leicht negative oder sehr negative Bilder, positive Bilder weniger. Diese Zusammenhänge müssen jedoch für andere Themen und andere Zielgruppen näher überprüft werden.

\section{Literatur}

Aust, C. F. \& Zillmann, D. (1996). Effects of victim exemplification in television news on viewer perception of social issues. Journalism E Mass Communication Quarterly, 73, 787-803.

Bonfadelli, H. \& Friemel, T. (2006). Kommunikationskampagnen im Gesundheitsbereich. Grundlagen und Anwendungen. Konstanz: UVK.

Boster, F. J. \& Mongeau, P. (1984). Fear-arousing persuasive messages. In: R. N. Bostrom \& B. H. Westley (Eds.), Communication Yearbook 8 (S. 330-375). Newbury Park, CA: Sage.

Brosius, H.-B. (1995). Alltagsrationalität in der Nachrichtenrezeption. Ein Modell zur Wabrnebmung und Verarbeitung von Nachrichteninhalten. Opladen: Westdeutscher Verlag.

Brosius, H.-B. \& Bathelt, A. (1994). The utility of exemplars in persuasive communications. Communication Research, 21, 48-78.

Brosius, H.-B., Schweiger, W. \& Rossmann, C. (2000). Auf der Suche nach den Ursachen des Fallbeispieleffekts: Der Einfluß von Art und Anzahl der Urheber von Fallbeispielinformation. Medienpsychologie, 12, 153-175.

Bundeszentrale für gesundheitliche Aufklärung (2007). Bilanzbericht 2004-2006. www.bzga.de/ botmed_96050000.html [25.6.2008].

Cegala, D. (2007). Interpersonale Kommunikation. In: J. Kerr, R. Weitkunat \& M. Moretti (Hrsg.), $A B C$ der Verbaltensänderung. Der Leitfaden für erfolgreiche Prävention und Gesundheitsförderung (S. 237-252). München: Urban \& Fischer. 
Daschmann, G. (2001). Der Einfluß von Fallbeispielen auf Leserurteile. Experimentelle Untersuchungen zur Medienwirkung. Konstanz: UVK.

Devos-Comby, L. \& Salovey, P. (2002). Applying persuasion strategies to alter HIV-relevant thoughts and behavior. Review of General Psychology, 6, 287-304.

Donovan, R. J. (2007). Zielgruppen und Zielverhalten. In: J. Kerr, R. Weitkunat \& M. Moretti (Hrsg.), ABC der Verhaltensänderung. Der Leitfaden für erfolgreiche Prävention und Gesundheitsförderung (S. 220-236). München: Urban \& Fischer.

Donovan, R. J., Egger, G. J. \& Francas, M. (1999). TARPARE: A method for selecting target audiences for public health interventions. Australian and New Zealand Journal of Public Health, 23, 280-284.

Easterling, D. V. \& Leventhal, H. (1989). Contribution of concrete cognition to emotion: Neutral symptoms as elicitors of worry about cancer. Journal of Applied Psychology, 74, 787-796.

Friend, K. \& Levy, D. T. (2002). Reductions in smoking prevalence and cigarette consumption associated with mass-media campaigns. Health Education Research, 17, 85-98.

Gibson, R. \& Zillmann, D. (1994). Exaggerated versus representative exemplification in news reports: Perception of issues and personal consequences. Communication Research, 21, 603624.

Glanz, K. (1985). Nutrition education for risk factor reduction and patient education: A review. Preventive Medicine, 14, 721-752.

Green, E. C. \& Witte, K. (2006). Can fear arousal in public health campaigns contribute to the decline of HIV prevalence? Journal of Health Communication, 11, 245-259.

Gregory, J. (2002). Social issues infotainment: Using emotion and entertainment to attract readers' attention in social issues leaflets. Information Design Journal, 11, 67-81.

Hawkey, G. M. \& Hawkey, C. J. (1989). Effect of information leaflets on knowledge in patients with gastrointestinal diseases. Gut, 30, 1641-1646.

Hoeken, H. \& Geurts, D. (2005). The influence of exemplars in fear appeals on the perception of self-efficacy and message acceptance. Information Design Journal and Document Design, 13, 238-248.

Hoeken, H. \& Hustinx, L. (2007). The impact of exemplars on responsibility stereotypes in fundraising letters. Communication Research, 34, 596-617.

Hovland, C. I., Janis, I. L. \& Kelley, H. H. (1953). Communication and persuasion: Psychological studies of opinion change. New Haven, CT: Yale University Press.

Hubert Burda Media (Hrsg.) (2006). Typologie der Wünsche 05/06. Methodenbeschreibung, Modelle, Grundzäblung. Offenburg: Burda Publishing Center.

Hunt, P. (1995). Development and evaluation of the 'Changing What You Eat' resources for primary care. Health Education Journal, 54, 405-414.

Hurrelmann, K., Klotz, T. \& Haisch, J. (Hrsg.) (2007). Lebrbuch Prävention und Gesundheitsförderung. Bern: Hans Huber.

Janis, S. \& Feshbach, S. (1953). Effects of fear-arousing communications. The Journal of Abnormal and Social Psychology, 48, 78-92.

Jazbinsek, D. (2000). Gesundheitskommunikation. Wiesbaden: Westdeutscher Verlag.

Job, R. F. S. (1988). Effective and ineffective use of fear in health promotion campaigns. American Journal of Public Health, 78, 163-167.

Kai, J. (1994). 'Baby Check' in the inner city - Use and value to parents. Family Practice, 11, 245250.

Keller, R. (2008). Wann wirken Spendenaufrufe? Der Einfluss von Bildauswabl und Argumentationsstruktur. München: R. Fischer.

Kohn, P. M., Goodstadt, M. S., Cook, G. M., Sheppard, M. \& Chan, G. (1982). Ineffectiveness of threat appeals about drinking and driving. Accident Analysis and Prevention, 14, 457-464.

Krisher, H. P., Darley, S. A. \& Darley, J. M. (1973). Fear-provoking recommendations, intentions to take preventive actions, and actual preventive actions. Journal of Personality and Social Psychology, 26, 301-308. 
Mensink, G. B. M., Lampert, T. \& Bergmann, E. (2005). Übergewicht und Adipositas in Deutschland 1984-2003. Bundesgesundheitsblatt, 48, 1348-1356.

Miller, G. R. (1963). Studies on the use of fear appeals: A summary and analysis. Central States Speech Journal, 24, 117-125.

Mongeau, P. (1998). Another look at fear arousing messages. In: M. Allen \& R. Press (Eds.), Persuasion: Advances through meta-analysis (S. 53-68). Cresskill, NJ: Hampton Press.

Naidoo, J. \& Wills, J. (2003). Lebrbuch der Gesundheitsförderung. Gamburg: Verlag für Gesundheitsförderung.

Närhi, U. (2007). Sources of medicine information and their reliability evaluated by medicine users. Pharmacy World E Science, 29, 688-694.

Nichols, S., Walters, W. E., Moolaway, M. \& Hamilton-Smith, M. B. (1988). Evaluation of the effectiveness of a nutritional health education leaflet in changing public knowledge and attitudes about eating and health. Journal of Human Nutrition and Dietetics, 1, 223-238.

o. V. (1997). Tips for planning your publication. PR News, 53, 1-2.

Ortony, A. \& Turner, T. J. (1990). What's basic about basic emotions? Psychological Review, 97, 315-331.

Petersen, T. (2006). Lasswells Frage und Hovlands Problem. Publizistik, 51, 39-51.

Robert Koch-Institut (Hrsg.) (2005). Übergewicht und Adipositas (Gesundheitsberichterstattung des Bundes, Heft 16). Berlin: Robert Koch-Institut.

Sutton, S. R. (1982). Fear-arousing communications: A critical examination of theory and research. In: J. R. Eiser (Ed.), Social psychology and behavioral medicine (S. 303-337). London: Wiley.

Thomas, J. (1994). New Approaches to Achieving Dietary Change. Current Opinion in Lipidology, $5,36-41$.

Vögele, C. (2007). Gesundheitsförderung und Gesundheitsprävention. In: J. Kerr, R. Weitkunat \& M. Moretti (Hrsg.), ABC der Verbaltensänderung. Der Leitfaden für erfolgreiche Prävention und Gesundheitsförderung (S. 293-312). München, Jena: Urban \& Fischer.

Welch Cline, R. J. (1990). Small group communication in health care. In: E. B. Ray \& L. Donohew (Eds.), Communication and health: Systems and applications (S. 69-91). Hillsdale, NJ: Erlbaum.

WHO (2007). Die Herausforderung Adipositas und Strategien zu ibrer Bekämpfung in der europäischen Region der WHO. Zusammenfassung: www.euro.who.int/document/E89858G.pdf? language $=$ German [23.6.2008].

Wirth, A. (2008). Adipositas. Ätiologie, Folgekrankheiten, Diagnose, Therapie. Heidelberg: Springer.

Witte, K. (1992). Putting the fear back into fear appeals: The extended parallel process model. Communication Monographs, 59, 329-349.

Witte, K. \& Allen, M. (2000). A meta-analysis of fear appeals: Implications for effective public health campaigns. Health Education \& Behavior, 27, 608-632.

Zillmann, D. (2006). Exemplification effects in the promotion of safety and health. Journal of Communication, 56, S221-S237.

Zillmann, D. \& Brosius, H.-B. (2000). Exemplification in communication: The influence of case reports on the perception of issues. Mahwah, NJ: Erlbaum.

Zillmann, D. \& Gan, S. (1996). Effects of threatening images in news programs on the perception of risk to others and self. Medienpsychologie, 8, 288-305.

Zillmann, D., Gibson, R. \& Sargent, S. L. (1999). Effects of photographs in news-magazine reports on issue perception. Media Psychology, 1, 207-228.

Zillmann, D., Gibson, R., Sundar, S. S. \& Perkins, J. W. (1996). Effects of exemplification in news reports on the perception of social issues. Journalism and Mass Communication Quarterly, 73, 427-444.

Zillmann, D., Knobloch, S. \& Yu, H. (2001). Effects of photographs on the selective reading of news reports. Media Psychology, 3, 301-324.

Zillmann, D., Perkins, J. W. \& Sundar, S. S. (1992). Impression-formation effects of printed news varying in descriptive precision and exemplifications. Medienpsychologie, 4, 168-185, 239240. 


\section{Anhang}

Schaubild A1: Vergleich der variierten Textpassagen

\begin{tabular}{|c|c|}
\hline Textversion „Fallbeispiel“ & $\begin{array}{l}\text { Textversion „summarische Realitäts- } \\
\text { beschreibung“ }\end{array}$ \\
\hline Adipositas kann jeden treffen & Adipositas in Zahlen und Fakten \\
\hline $\begin{array}{l}\text { Sabine H. ist übergewichtig. Bei einer Grö- } \\
\text { Be von } 1,65 \mathrm{~m} \text { wiegt sie } 76 \text { Kilogramm: Ihr } \\
\text { Body-Mass-Index (BMI) hat somit einen } \\
\text { Wert von 27,9. Dass das zu viel ist und } \\
\text { negative Folgen für ihre Gesundheit hat, ist } \\
\text { der 39-Jährigen bewusst. }\end{array}$ & $\begin{array}{l}\text { Über die Hälfte der Deutschen ist übergewich- } \\
\text { tig. Das heißt, der Body-Mass-Index (BMI) der } \\
\text { Betroffenen hat einen Wert von über 25. Das ist zu } \\
\text { viel und kann negative Folgen für die Gesundheit } \\
\text { haben. }\end{array}$ \\
\hline $\begin{array}{l}\text { Übergewicht und Adipositas zählen zu den } \\
\text { häufigsten ernährungsbedingten Gesund- } \\
\text { heitsstörungen in Deutschland: } 55 \% \text { der } \\
\text { Frauen und über } 65 \% \text { der Männer haben } \\
\text { einen BMI über } 25 . \text { Die Gefahr der Adipo- } \\
\text { sitas steigt mit zunehmendem Alter. (nicht } \\
\text { variiert) }\end{array}$ & $\begin{array}{l}\text { Übergewicht und Adipositas zählen zu den } \\
\text { häufigsten ernährungsbedingten Gesundheitsstö- } \\
\text { rungen in Deutschland: } 55 \% \text { der Frauen und über } \\
65 \% \text { der Männer haben einen BMI über 25. Die } \\
\text { Gefahr der Adipositas steigt mit zunehmendem } \\
\text { Alter. }\end{array}$ \\
\hline $\begin{array}{l}\text { „Als Jugendliche war ich immer normalge- } \\
\text { wichtig“, berichtet Sabine H. Doch durch } \\
\text { Stress im Job habe sie später immer häufi- } \\
\text { ger zu ungesunden Lebensmitteln gegriffen: } \\
\text { Fast Food, wenn es schnell gehen musste; } \\
\text { Süßigkeiten, wenn die Laune wieder einmal } \\
\text { im Keller war. }\end{array}$ & $\begin{array}{l}\text { Als Jugendliche sind die Betroffenen oft noch } \\
\text { normalgewichtig. Doch durch Stress, z. B. im } \\
\text { Job, wird vermehrt zu ungesunden Lebensmitteln } \\
\text { gegriffen: Fast Food, wenn es schnell gehen muss; } \\
\text { Süßigkeiten, wenn die Laune wieder einmal im } \\
\text { Keller ist. }\end{array}$ \\
\hline $\begin{array}{l}\text { „Durch die Geburt meines ersten Kindes } \\
\text { habe ich auch immer weniger Sport getrie- } \\
\text { ben“, so die inzwischen zweifache Mutter. } \\
\text { Die Folgen sind offensichtlich: Sabine H. } \\
\text { leidet an Gelenkschäden, Bluthochdruck } \\
\text { und hat Herz-Kreislauf-Beschwerden. } \\
\text { Auch psychische Probleme hat das Überge- } \\
\text { wicht mit sich gebracht: „Seit Jahren traue } \\
\text { ich mich wegen der Dehnungsstreifen nicht } \\
\text { mehr ins Schwimmbad. Mein Selbstbe- } \\
\text { wusstsein ist deutlich geringer geworden.“ }\end{array}$ & $\begin{array}{l}\text { Aufgrund von Kindern oder anderen familiären } \\
\text { Verpflichtungen wird oftmals weniger Sport } \\
\text { getrieben. Mögliche Folgen sind: Gelenkschäden, } \\
\text { Bluthochdruck, Herz-Kreislauf-Beschwerden, } \\
\text { aber auch psychische Probleme kann das Überge- } \\
\text { wicht mit sich bringen. Gerade die sog. Striae, also } \\
\text { Dehnungsstreifen am ganzen Köper, beeinträchti- } \\
\text { gen das Selbstwertgefühl. Die Betroffen trauen sich } \\
\text { nicht mehr ins Schwimmbad. Das Selbstbewusst- } \\
\text { sein wird deutlich geringer. }\end{array}$ \\
\hline $\begin{array}{l}\text { Eine nachhaltige Ernährungsumstellung } \\
\text { und körperliche Betätigung können helfen. } \\
\text { (nur marginal variiert) }\end{array}$ & $\begin{array}{l}\text { Eine nachhaltige Ernährungsumstellung und kör- } \\
\text { perliche Betätigung wirken dem entgegen. }\end{array}$ \\
\hline $\begin{array}{l}\text { Tipps zu Essgewohnheiten, Ernährung } \\
\text { und Bewegung finden Sie umseitig. (nicht } \\
\text { variiert) }\end{array}$ & $\begin{array}{l}\text { Tipps zu Essgewohnheiten, Ernährung und Bewe- } \\
\text { gung finden Sie umseitig. }\end{array}$ \\
\hline
\end{tabular}




\section{Schaubild A2: $\quad$ Überblick über die abgefragten Merkmale und ibre Operationalisierung}

\section{Abhängige Variablen}

Wissen
$\left(t_{1}\right.$ und $\left.t_{2}\right)$

Risikowahrnehmung $\left(\mathrm{t}_{1}\right.$ und $\left.\mathrm{t}_{2}\right)$

Einstellungen zu Ernährung und Übergewicht $\left(t_{1}\right.$ und $\left.t_{2}\right)$
Verhaltensintention $\left(\mathrm{t}_{1}\right)$
Definition BMI: geschlossene Abfrage, zwei Fragen mit richtigen und falschen Antwortmöglichkeiten $\rightarrow$ summarischer Index der richtigen abzüglich der falschen Antworten

Folgeerkrankungen: geschlossene Abfrage, neun richtige und drei falsche Folgeerkrankungen $\rightarrow$ summarischer Index der richtigen abzüglich der falschen Antworten

Prophylaxe: geschlossene Abfrage, acht Statements (z. B. „Durch körperliche Betätigung kann man langfristig abnehmen.“; „Nur durch strikte Nulldiät ist es möglich abzunehmen.") $\rightarrow$ summarischer Index der richtigen abzüglich der falschen Antworten

Wissen Gesamt: summarischer Index aus den Indizes BMI, Folgen und Prophylaxe

Anteil Übergewichtiger: offene Frage „Was glauben Sie, wie viele Menschen in Deutschland sind übergewichtig?“"

Anteil Kranker: offene Frage „Was glauben Sie, wie viele Menschen in Deutschland haben aufgrund von Übergewicht gesundheitliche Probleme?“ Risiko zu erkranken: „Was glauben Sie, wie hoch ist das Risiko für Sie, aufgrund von Übergewicht an den folgenden Krankheiten zu erkranken?" (fünfstufige Antwortskala: „sehr niedrig“, „eher niedrig“, „mittel“, „eher hoch“, „hoch“; Items: „Herz-Kreislaufprobleme“, „Gelenkschäden“, „psychische Belastungen" $\rightarrow$ summarischer Index)

Sorge zu erkranken: „Wie häufig machen Sie sich Sorgen, aufgrund von Übergewicht an diesen Krankheiten zu erkranken?" (Skala und Items vgl. Risiko zu erkranken)

Fünfstufige Likertskala („trifft überhaupt nicht zu“ bis „trifft voll und ganz $\mathrm{zu}$ “), zehn Items:

Gesamtindex nicht reliabel

$\mathrm{t}_{1}$ : Faktorenanalyse (Varimax-Rotation, Kaiserkriterium): vier Faktoren (60\% erklärte Varianz):

„Genussorientierung“: zwei Items, z. B. „Essen ist für mich eines der größten Vergnügen.“; „Gesundheitsbewusstsein“: drei Items, z. B. „Es ist wichtig auf eine gesunde, ausgewogene Ernährung zu achten.“; „Gelassenheit“: drei Items, z. B. „Attraktivität hat nichts mit Körpergewicht zu tun.“; „Gewichtsbewusstsein“: zwei Items, „Ich bin oft neidisch, wenn ich jemanden mit einer guten Figur sehe.“, „Es ist wichtig beim Essen auf Kalorien zu achten.“

$\mathrm{t}_{2}$ : Faktorenanalyse: keine sinnvolle Lösung; nicht mit $\mathrm{t}_{1}$ vergleichbar, daher wird hier je Dimension der Faktorenlösung $\left(t_{1}\right)$ stellvertretend ein Item herangezogen (jeweils das Item, welches bei der Faktorenanalyse von $t_{1}$ am stärksten auf der jeweiligen Dimension lädt, siehe Beispiele bei $\mathrm{t}_{1}$ ).

„Wie sehen Ihre eigenen Pläne bezüglich Ernährung und Sport aus?“, fünfstufige Likertskala: „stimme überhaupt nicht zu“ bis „stimme voll und ganz zu“, Residualantwort „tue ich bereits“, neun Items, z. B. „Ich habe vor, eine Ernährungsberatungsstelle aufzusuchen.", „Ich habe vor, in Zukunft öfter die Treppe dem Lift vorzuziehen."

$\rightarrow$ Mittelwertindex (Cronbach’s Alpha: 0,85) 


\begin{tabular}{|c|c|}
\hline \multicolumn{2}{|c|}{ Abhängige Variablen (Fortsetzung) } \\
\hline $\begin{array}{l}\text { Verhalten in den } \\
\text { vergangenen } \\
\text { zwei Wochen } \\
\left(\mathrm{t}_{2}\right)\end{array}$ & $\begin{array}{l}\text { „Wie sah Ihr Ernährungs- und Bewegungsverhalten in den letzten zwei } \\
\text { Wochen aus?“, fünfstufige Likertskala: „trifft überhaupt nicht zu“ bis „trifft } \\
\text { voll und ganz zu“, neun Items äquivalent zu Verhaltensintention, z. B. } \\
\text { „Ich habe eine Ernährungsberatungsstelle aufgesucht.“, „Ich habe öfter die } \\
\text { Treppe dem Lift vorgezogen.“ } \\
\rightarrow \text { Mittelwertindex (Cronbach’s Alpha: } 0,77 \text { ) }\end{array}$ \\
\hline \multicolumn{2}{|c|}{ Intervenierende Variablen } \\
\hline $\begin{array}{l}\text { Soziodemogra- } \\
\text { phie }\left(t_{1}\right)\end{array}$ & Alter (offen), Geschlecht, Bildung (höchster Bildungsabschluss) \\
\hline $\operatorname{BMI}\left(t_{1}\right)$ & Körpergröße (offen), Gewicht (offen) \\
\hline $\begin{array}{l}\text { physische Akti- } \\
\text { vität }\left(t_{1}\right)\end{array}$ & Sport pro Woche (offen in Stunden und Minuten) \\
\hline $\begin{array}{l}\text { Nutzung Ge- } \\
\text { sundheitsflyer } \\
\left(\mathrm{t}_{1}\right)\end{array}$ & $\begin{array}{l}\text { „Wenn Sie an die letzten vier Wochen denken, wie viele Gesundheitsflyer } \\
\text { haben Sie sich in dieser Zeit angesehen?"“ (vierstufig: „keinen“, „1 bis 2“, „3 } \\
\text { bis } 4 \text { “ und „5 und mehr“) }\end{array}$ \\
\hline Involvement $\left(t_{1}\right)$ & $\begin{array}{l}\text { Fünfstufige Likertskala, sechs Items (z. B. „Alles in allem bin ich mit mei- } \\
\text { nem Körper zufrieden.“, „Eigentlich sollte ich ein paar Kilo abnehmen.“) } \\
\rightarrow \text { Mittelwertindex (Cronbach’s Alpha: 0,86) }\end{array}$ \\
\hline $\begin{array}{l}\text { Emotionale } \\
\text { Reaktionen }\left(t_{1}\right)\end{array}$ & $\begin{array}{l}\text { Fünfstufige Likertskala, } 15 \text { Items } \\
\text { Einzeldimensionen (theoretisch hergeleitet): „Betroffenheit“: } 10 \text { Items, } \\
\text { z. B. „Die geschilderte Situation nimmt mich persönlich mit“ (Cronbach’s } \\
\text { Alpha: 0,85), „Mitleid“: drei Items, z. B. „Adipöse Menschen tun mir leid.“ } \\
\text { (Cronbach’s Alpha: 0,67) } \\
\rightarrow \text { Gesamtindex (Cronbach’s Alpha: 0,83) }\end{array}$ \\
\hline \multicolumn{2}{|c|}{ Treatment-Check } \\
\hline $\begin{array}{l}\text { Bewertung des } \\
\text { Flyers }\left(t_{1}\right)\end{array}$ & $\begin{array}{l}\text { Semantisches Differenzial, fünfstufig, } 13 \text { Items, z. B. „vertrauenswürdig / } \\
\text { suspekt“, „sachlich / emotional“ } \rightarrow \text { Mittelwertindex (Cronbach’s Alpha: } \\
\text { 0,86) }\end{array}$ \\
\hline $\begin{array}{l}\text { Wahrnehmung } \\
\text { des Fotos }\left(\mathrm{t}_{1}\right)\end{array}$ & $\begin{array}{l}\text { Fünfstufige Likertskala, elf Items, drei Dimensionen nach Faktorenanalyse } \\
\text { (Varimax-Rotation, Kaiserkriterium), } 62 \text { \% erklärte Varianz: „Betrof- } \\
\text { fenheit“: fünf Items, z. B. „Das Foto ist ein typisches Krankheitsbild“; } \\
\text { „Ablehnung“: drei Items, z. B. „Das Foto erweckt Abneigung, da ändert } \\
\text { man schon aus Prinzip nichts“; „Nüchtern“: zwei Items, z. B. „Das ist ein } \\
\text { typisches Foto für einen Gesundheitsflyer. Die Fotos zu anderen Gesund- } \\
\text { heitsthemen sehen genauso aus.“ }\end{array}$ \\
\hline
\end{tabular}

